\title{
Non-Smoothness of Event Horizons of Robinson-Trautman Black Holes
}

\author{
Piotr T. Chruściel` and David B. Singleton ${ }^{\star \star}$ \\ Australian National University, G.P.O. Box 4, Canberra A.C.T. 2601, Australia
}

Received July 1, 1991

\begin{abstract}
It is shown that generic "small data" Robinson-Trautman space-times cannot be $C^{123}$ extended beyond the " $r=2 m$ Schwarzschild-like" event horizon. This implies that an observer living in such a space-time can determine by local measurements whether or not he has crossed the event-horizon of the black-hole.
\end{abstract}

\section{Introduction}

Perhaps the two most striking predictions of Einstein's theory of gravitation are the existence of gravitational radiation and of black holes. There are known four classes of asymptotically flat space-times containing gravitational radiation, the global structure of which is reasonably well understood: the Christodoulou-Klainerman metrics [7], the Friedrichs metrics [13], the boost-rotation symmetric metrics [2] and $^{1}$ the Robinson-Trautman (RT) metrics [17]. On the other hand known examples of space-times which contain a black hole are given by the Kerr-Newman space-times, the static Einstein-Maxwell Majumdar-Papapetrou multi-black hole solutions, the Tolman-Bondi perfect fluid metrics, Christodoulou's collapsing scalar field black-holes [6] (for these last two classes of space-times the metric in the vacuum region is the Schwarzschild metric) and the RT space-times. The privileged role of the Robinson-Trautman space-times stems from the fact that they provide an arena in which both gravitational radiation and black-hole formation can be studied simultaneously, in the vacuum. These space-times were orignally

\footnotetext{
* Centre for Mathematics and its Applications of the ANU; on leave of absence from the Institute of Mathematics, Polish Academy of Sciences, Warsaw. e-mail: cmc851@csc.anu.edu.au

$\star \star$ Department of Mathematics of the ANU. e-mail: dbs661@csc2.anu.edu.au

${ }^{1}$ It seems that Christodoulou's scalar field space-times $[5,6]$ should not be considered as containing gravitational radiation, since by Birkhoff's theorem the metric is the Schwarzschild one wherever the scalar field $\phi$ vanishes. Moreover, the $1 / r$ part of the Riemann tensor, usually thought of as the manifestation of gravitational radiation, vanishes for these metrics (D. Christodoulou, private communication)
} 
discovered in a search for metrics containing gravitational radiation [17], and it is only recently that it has been recognized that the RT metrics can be used as building blocks for constructing black-hole space-times: in Ref. [21] it was shown that any two RT space-times can be "glued" together along a Schwarzschild-type " $r=2 m$ " event horizon to form a space-time which contains both a black and a white hole ${ }^{2}$, with global structure somewhat similar to that of the Kruskal-Szekeres extensions of the $r>2 m$ Schwarzchild space-time. Although not explicitly stated in [21], the space-times so constructed were generically expected to have a metric of $C^{5}$ but not $C^{6}$ differentiability class (cf. also [9, 19, 20]). It was shown in [9] that a careful choice of the space-times which were being glued together led to a space-time the metric of which was of at least $C^{117}$ differentiability class, and the methods of proof of that paper suggested very strongly that for generic RT space-times no extensions beyond the " $r=2 m$ event horizon" with a metric of $C^{118}$ differentiability class will exist. In this paper we show that generic RT space-times evolving from "sufficiently small" initial data admit no $C^{123}$ extensions, vacuum or otherwise, across the " $r=2 m$ " null boundary. We believe that generic RT space-times do not admit extensions with a metric of $C^{118}$ differentiability class; thus the small-data restriction is probably not necessary, while the discrepancy between $C^{123}$ and $C^{118}$ is an artefact due to the inextendability criterion used.

It may be argued that a singularity which shows up in the $118^{\prime}$ th (or $123^{\prime} \mathrm{rd}$ ) derivatives of the metric has no physical meaning, and that anything which is $C^{k}$ with $k \geqq 2$ may be considered as being smooth, as far as physical applications are concerned. We believe that this is not the case. For instance, an observer in a space-time with a smooth event-horizon has no way of detecting by local measurements whether or not he has crossed the event horizon, while an observer in a Robinson-Trautman space-time with a singular horizon can in principle keep track of the $120^{\prime}$ th derivatives of the scalar $\nabla^{v} R^{\alpha \beta \gamma \delta} \nabla_{v} R_{\alpha \beta \gamma \delta}$ and verify, by observing their blow up, that he has entered the region from which he can no longer communicate with the outside world. This unexpected property of generic RobinsonTrautman black-holes should probably be considered as a manifestation of the naked singularity $r=0$, since the metric in space-times evolving from smooth data on a spacelike Cauchy surface, in which a stable version of cosmic censorship holds, is necessarily smooth in a neighbourhood of the event horizon.

This paper is organized as follows: in Sect. 2 we briefly review what is known about solutions of the RT equation, and give the precise statement of our main results, Theorems 2.1 and 2.2. A "final state" characterization of those RT space-times for which the event horizon $\mathscr{H}$ is singular is presented in Sect. 2.1 when $m>0,{ }^{2} \mathscr{M}=S^{2}$, and in Sect. 2.2 for $m<0,{ }^{2} \mathscr{M} \neq S^{2}, T^{2}$, where $S^{2}$ is the two-dimensional sphere and $T^{2}$ is the two-dimensional torus. In Sect. 3.1 results on the linearized RT equation needed for the proofs of Theorems 2.1 and 2.2 are established; and the proofs of Theorems 2.1 and 2.2 are given in Sect. 3.2.

\footnotetext{
${ }^{2}$ In the "maximally extended" RT space-times, as considered in Sect. 2, the event horizon can be defined as usual as the boundary of the past of $\mathcal{I}^{+}:$this justifies the statement of the existence of a black hole. On the other hand the notion of the "white hole" in these space-time is only an intuitive one (cf. also [21] for a discussion), since generic RT space-times cannot be extended up to $\mathcal{I}^{+}$(in the RT class of vacuum metrics; cf. [8] [Proposition 2.1])
} 


\section{A "Final State" Characterization of Robinson-Trautman Space-Times with a Singular " $r=2 m$ " Horizon}

Let $\stackrel{\circ}{a b}_{a b}$ be a smooth metric on a two dimensional, compact, connected, orientable manifold ${ }^{2} \mathscr{M}$, let $f(u)$ be a $u$-dependent family of positive functions on ${ }^{2} \mathscr{M}$. It has been shown by Robinson and Trautman [17] that if the $u$-dependent family of metrics

$$
g_{a b}=f(u)^{-2} \stackrel{\circ}{g}_{a b}
$$

satisfies the evolution equation

$$
\frac{\partial g_{a b}}{\partial u}=\frac{1}{12 m} \Delta_{g} R g_{a b}
$$

where $m$ is a constant, $R(g)=R_{a b}^{a b}$ is the curvature scalar of the metric $g_{a b}$ and $\Delta_{g}$ $\left(\Delta_{o}\right)$ denotes the Laplacian of the metric $g(g)$, then the four-dimensional Lorentzian metric

$$
\begin{gathered}
d s^{2}=-\Phi d u^{2}-2 d u d r+r^{2} f^{-2} \stackrel{\circ}{g}_{a b} d x^{a} d x^{b}, \\
\Phi=\frac{R}{2}+\frac{r}{12 m} \Delta_{g} R-\frac{2 m}{r}, \quad R \equiv R(g),
\end{gathered}
$$

will satisfy the vacuum Einstein equations. Equation (2.2) is a quasi-linear parabolic equation for $f$,

$$
\begin{aligned}
\frac{\partial f}{\partial u} & =-\frac{f}{24 m} \Delta_{g} R, \\
R & =R(g)=f^{2}\left(R_{o}+2 \Delta_{o} \ln f\right), \\
\Delta_{g} & =f^{2} \Delta_{o},
\end{aligned}
$$

where $R_{o}$ is the curvature scalar of the metric $\stackrel{\circ}{g}_{a b}$. Solutions of (2.4) can be found by prescribing $f\left(u_{o}\right) \in H_{4+k}\left({ }^{2} \mathscr{M}\right), k \geqq 0$ and integrating forward in $u$ if $m>0$ or backward in $u$ if $m<0\left(H_{l}\left({ }^{2} \mathscr{M}\right)\right.$ is the Hillbert space of functions the derivatives of which up to order $l$ are square integrable on ${ }^{2} \mathscr{M}$ ). Local existence of solutions of this problem was first pointed out in the physical literature by Schmidt [18]; existence for all $u \geqq u_{o}$ with "small initial data" has been shown by Rendall [16] when ${ }^{2} \mathscr{M} \neq S^{2}$ ( $S^{2}$ denotes the two dimensional sphere), and in [20] when ${ }^{2} \mathscr{M}=S^{2}$; existence for all $u \geqq u_{o}$ without restrictions on the size of the data has been shown in [8]. In that last reference it has also been shown that every solution of (2.4) immediately becomes smooth (in fact, even analytic). In [9] it has been shown that there exists a sequence $N(i)$, with $N(0)=N(1)=0$, and a strictly increasing sequence $\left\{v_{i}\right\}, v_{o}=0$, depending only upon the metric $\stackrel{\circ}{a b}_{a b}$, such that every solution of $(2.4)$ has an expansion of the form

$$
\forall n \in \mathbb{N} \quad f=\sum_{i=0}^{n} \sum_{j=0}^{N} f_{i, j} u^{j} e^{-v_{i} u}+r_{n}
$$

with some ( $u$-independent) functions $f_{i, j} \in C^{\infty}\left({ }^{2} \mathscr{M}\right)$, and

$$
\forall i, k \in \mathbb{N}, \quad u \geqq u_{o}+1, \quad\left|\stackrel{\circ}{\nabla}^{k} \frac{\partial^{i}}{(\partial u)^{i}} r_{n}\right| \leqq C_{n, k, i} u^{N(n+1)} e^{-v_{n+1} u},
$$




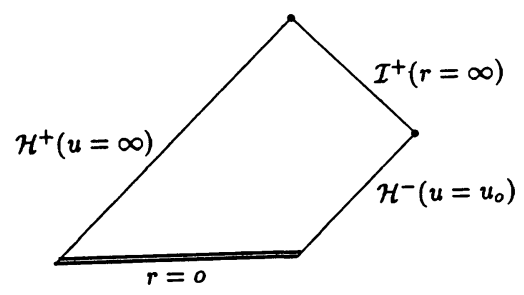

Fig. 2.1. $m>0$

where $\stackrel{\circ}{\nabla}$ is the covariant Riemannian derivative of the metric $\stackrel{\circ}{g}_{a b}$, for some constants $C_{n, k, i}$ depending upon the solution $f$.

2.1. ${ }^{2} \mathscr{M}=S^{2}$. Conformally rescaling the metric $\stackrel{\circ}{g}_{a b}$ and redefining $f\left(u_{o}\right)$ if necessary we can without loss of generality assume that $\stackrel{\circ}{a b}_{a b}$ is the standard "round metric" on the sphere, $R\left(\stackrel{\circ}{a b}_{a b}\right)=2$. In that case $f_{0,0}$ in the expansion (2.6) can be set to 1 by an appropriate conformal transformation, and we also have

$$
\begin{array}{rlrl}
v_{i} & =v \times i, & v & =2 / m, \\
0 \leqq i \leqq 14, & N(i) & =0, \\
i & =15, & N(i) & =1,
\end{array}
$$

(cf. [9] [Proposition 4.2]). The expansion (2.6) in the $S^{2}$ case thus takes the form

$$
f=1+f_{1} e^{-v u}+f_{2} e^{-2 v u}+\cdots+f_{14} e^{-14 v u}+f_{\log } u e^{-15 v u}+f_{15} e^{-15 v u}+\cdots
$$

Given a solution of (2.4) defined on $\left[u_{o}, \infty\right)$ the corresponding space-time ${ }^{4} \mathscr{M}$ with $m>0$ has the global structure displayed in Fig. 2.1, and it has been showed by Tod [21] that the space-time ${ }^{4} \mathscr{M}$ can be extended across $\mathscr{H}^{+}$in a way similar to the Kruskal-Szekeres extension of the $r>2 m$ Schwarzschild space-time (cf. also [9]) (a black-hole - white-hole space-time with a metric of $C^{117}$ differentiability can be obtained by glueing to ${ }^{4} \mathscr{M}$ a time-reversed, space-inverted copy of itself along $\mathscr{H}^{+}$, as one does in the Kruskal-Szekeres-Schwarzschild manifold). The main result of our paper is the following:

Theorem 2.1. There exists an open nonempty subset $X$ of $C^{\infty}\left({ }^{2} \mathscr{M}\right)$ such that if $f\left(u_{o}\right) \in X$ then the corresponding RT space-time cannot be extended across $\mathscr{H}^{+}$in the class of manifolds with $C^{123}$ Lorentzian, vacuum or otherwise, metrics. Moreover there exists $\varepsilon_{o}>0$ such that the set $B_{\varepsilon_{o}} \cap X$ is dense in $B_{\varepsilon_{o}} \cap C^{\infty}\left({ }^{2} \mathscr{M}\right)$ equipped with a $C^{\infty}\left({ }^{2} \mathscr{M}\right)$ topology, where $B_{\varepsilon}=\left\{f\left(u_{o}\right) \stackrel{\varepsilon_{o}}{\in} H_{6}\left({ }^{2} \mathscr{M}\right):\left\|\ln f\left(u_{o}\right)\right\|_{H_{6}\left({ }^{2} \mathscr{M}\right)} \leqq \varepsilon\right\}$.

We have stated Theorem 2.1 in a $C^{\infty}$ setting to emphasize the fact that the non-differentiability of the extensions across $\mathscr{H}^{+}$has nothing to do with the potentially low differentiability of the initial data $f\left(u_{o}\right)$. In fact we also have the following stronger statement:

Theorem 2.2. Let $k \in \mathbb{N}, k \geqq 4$. There exists an open nonempty subset $X_{k}$ of $H_{k}\left({ }^{2} \mathscr{M}\right)$ such that if $f\left(u_{o}\right) \in X_{k}$ then the corresponding $\mathrm{RT}$ space-time cannot be extended across $\mathscr{H}^{+}$in the class of manifolds with $C^{123}$ Lorentzian, vacuum or otherwise, metrics. Moreover for $k \geqq 6$ there exists $\varepsilon_{o}>0$ such that $B_{\varepsilon_{o}} \cap X_{k}$ is dense in 
$B_{\varepsilon_{o}} \cap H_{k}\left({ }^{2} \mathscr{M}\right)$ (equipped with a $H_{k}\left({ }^{2} \mathscr{M}\right)$ topology), where $B_{\varepsilon}$ is defined in the statement of Theorem 2.1.

To prove Theorems 2.1 and 2.2 we shall need several auxiliary results, some of which are of independent interest; the proofs of Theorems 2.1 and 2.2 are deferred to Sect. 3. Let us start with the following statement:

Lemma 2.1. Consider the expansion (2.8):

1. $f_{1}$ is a linear combination with constant coefficients of $l=2$ spherical harmonics $\phi_{\alpha}^{+}$

$$
\begin{aligned}
f_{1} & =\sum_{\alpha} B_{\alpha}^{+} \phi_{\alpha}^{+}, B_{\alpha}^{+} \in \mathbb{R}^{5}, \\
\Delta_{o} \phi_{\alpha}^{+} & =-6 \phi_{\alpha}^{+} .
\end{aligned}
$$

2. Let $P$ denote the antipodal map of the sphere into itself. For $i=1, \ldots, 4$ we have

$$
f_{i} \circ P=f_{i} .
$$

3. There exists a homogeneous polynomial $\psi\left(B_{\alpha}^{+}\right)$of degree 5 in $B_{\alpha}^{+}$with coefficients being smooth antipodally symmetric functions on $S^{2}$ such that $f_{5}-\psi$ is a linear combination with constant coefficients of $l=3$ spherical harmonics $\phi_{\alpha}^{-}$,

$$
\begin{aligned}
f_{5} & =\psi\left(B_{\alpha}^{+}\right)+\sum_{\alpha} B_{\alpha}^{-} \phi_{\alpha}^{-}, \quad B_{\alpha}^{-} \in \mathbb{R}^{7}, \\
\Delta_{o} \phi_{\alpha}^{-} & =-12 \phi_{\alpha}^{-} .
\end{aligned}
$$

If $B_{\alpha}^{-}=0$ then (2.9) holds for $1 \leqq i \leqq 34$.

4. Set $\left(C_{\beta}\right)=\left(B_{\alpha}^{+},\left(B_{\alpha}^{-}\right)^{1 / 5}\right) \in \mathbb{R}^{12}$. There exist homogeneous polynomials $E_{\gamma}\left(C_{\beta}\right)$ of degree 15 with constant coefficients such that $f_{\log }$ is a linear combination of $l=4$ spherical harmonics $\chi_{\gamma}$ with coefficients $E_{\gamma}$ :

$$
\begin{aligned}
& f_{\log }=\sum_{\gamma} E_{\gamma}\left(C_{\beta}\right) \chi_{\gamma}, \\
& \Delta_{o} \chi_{\gamma}=-20 \chi_{\gamma} .
\end{aligned}
$$

Proof. Equation (2.4) can be rewritten in the form

$$
\frac{\partial f}{\partial u}=-\frac{f^{4}}{12 m}\left(\Delta_{o}^{2} f+2 \Delta_{o} f\right)+\frac{f^{3}}{12 m}\left[2 \stackrel{\circ}{\nabla}^{a b} f \stackrel{\circ}{\nabla}_{a b} f-\left(\Delta_{o} f\right)^{2}\right] .
$$

Inserting the expansion (2.8) in (2.11) one finds the following hierarchy of equations:

$$
1 \leqq i \leqq 14, \quad L_{i} f_{i} \equiv[L+i v] f_{i}=g_{i}\left(f_{1}, \ldots, f_{i-1}\right)
$$

with

$$
L=-\frac{1}{12 m}\left(\Delta_{o}^{2}+2 \Delta_{o}\right)
$$

The $g_{i}$ 's are obtained by grouping together terms containing the exponent $e^{-i v u}$ in the right-hand side of the equation

$$
\sum_{i=0}^{\infty} g_{i} e^{-i v u}=\frac{f^{4}-1}{12 m}\left(\Delta_{o}^{2} f+2 \Delta_{o} f\right)-\frac{f^{3}}{12 m}\left[2 \stackrel{\circ}{\nabla}^{a b} f \stackrel{\circ}{\nabla}_{a b} f-\left(\Delta_{o} f\right)^{2}\right]
$$


so that one has $g_{o}=g_{1}=0$. A simple REDUCE code gives

$$
\begin{aligned}
g_{2}= & \frac{1}{m}\left(8 f_{1}^{2}-Q\left(f_{1}, f_{1}\right)\right), \\
g_{3}= & -\frac{1}{m}\left(20 f_{1}^{3}-24 f_{1} f_{2}+2 Q\left(f_{1}, f_{2}\right)-f_{1} Q\left(f_{1}, f_{1}\right)\right), \\
g_{4}= & \frac{1}{m}\left(40 f_{1}^{4}-80 f_{1}^{2} f_{2}+32 f_{1} f_{3}+16 f_{2}^{2}-2 Q\left(f_{1}, f_{3}\right)-Q\left(f_{2}, f_{2}\right)\right. \\
& \left.+2 f_{1} Q\left(f_{1}, f_{2}\right)+f_{2} Q\left(f_{1}, f_{1}\right)-f_{1}^{2} Q\left(f_{1}, f_{1}\right)\right), \\
g_{5}= & -\frac{1}{m}\left(70 f_{1}^{5}-200 f_{1}^{3} f_{2}+100 f_{1}^{2} f_{3}-40 f_{1} f_{4}+100 f_{1} f_{2}^{2}-40 f_{2} f_{3}\right. \\
& +2 Q\left(f_{1}, f_{4}\right)+2 Q\left(f_{2}, f_{3}\right)-2 f_{1} Q\left(f_{1}, f_{3}\right)-f_{3} Q\left(f_{1}, f_{1}\right)-f_{1} Q\left(f_{2}, f_{2}\right) \\
& \left.-2 f_{2} Q\left(f_{1}, f_{2}\right)+2 f_{1}^{2} Q\left(f_{1}, f_{2}\right)+2 f_{1} f_{2} Q\left(f_{1}, f_{1}\right)-f_{1}^{3} Q\left(f_{1}, f_{1}\right)\right),
\end{aligned}
$$

etc., where

$$
Q\left(f_{i}, f_{j}\right) \equiv \frac{1}{12}\left(2 \stackrel{\circ}{\nabla}^{a b} f_{i} \stackrel{\circ}{\nabla a b}_{a} f_{i}-\Delta_{o} f_{i} \Delta_{o} f_{j}\right),
$$

and we have used the equations satisfied by the $f_{i}$ 's to somewhat simplify the expressions for the $g_{i}$ 's. The eigenfunctions of $L$ are the spherical harmonics and its

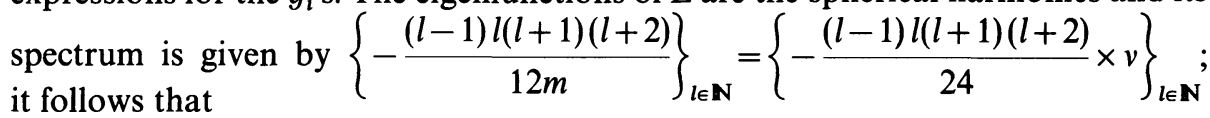

1. $f_{1}$ is in the kernel of $L_{1}$ and is thus a linear combination of $l=2$ spherical harmonics.

2. For $2 \leqq i \leqq 14, i \neq 5$, the operators $L_{i}$ have trivial kernels and thus Eq. (2.12) can be solved uniquely for $f_{i}$ in terms of $g_{i}$.

3. Since $L$ commutes with $P$, where $P$ is the antipodal map of the sphere into itself, one can show by induction (cf. e.g. [9] [Proposition 4.2]) that for $1 \leqq i \leqq 4$ one has $g_{i} \circ P=g_{i}, f_{i} \circ P=f_{i}$ and also $g_{5} \circ P=g_{5}$.

4. $L_{5}$ has a non-trivial kernel consisting of $l=3$ spherical harmonics; this implies that Eq. (2.12) with $i=5$ has the integrability conditions

$$
\int_{S^{2}} g_{5} \psi_{\sigma} d \mu_{o}=0
$$

where the $\psi_{\sigma}$ are $l=3$ spherical harmonics and $d \mu_{o}$ is the standard $S O(3)$ invariant measure on $S^{2}$. This is automatically satisfied because $g_{5}$ has even parity $\left(g_{5} \circ \boldsymbol{P}=g_{5}\right)$ while $\psi_{\sigma} \circ \boldsymbol{P}=-\psi_{\sigma}$. We can therefore solve for $f_{5}$ which is then defined up to the addition of $l=3$ spherical harmonics.

5. For $i=15$ one finds

$$
\begin{gathered}
L_{15} f_{\text {log }}=0, \\
L_{15} f_{15} \equiv[L+15 v] f_{15}=g_{15}\left(f_{1}, \cdots, f_{14}\right)+f_{\text {log }} .
\end{gathered}
$$

The kernel of $L_{15}$ consists of $l=4$ spherical harmonics $\chi_{\gamma}$, so that $f_{\log }$ must be a 
linear combination of those. The integrability conditions of (2.15) read

$$
\int_{S^{2}}\left(g_{15}+f_{\log }\right) \chi_{\gamma} d \mu_{o}=0
$$

which determines $f_{\log }$ uniquely in terms of $f_{1}$ and $f_{5}$.

6. The functions $g_{i}$ are polynomials in $f_{j}$ and their derivatives by construction, which

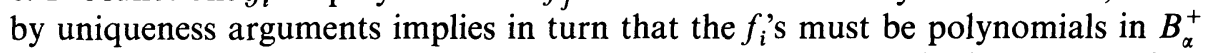
and $B_{\alpha}^{-}$. The homogeneity of order $i$ of $g_{i}$ and of $f_{i}$ follows easily by construction, but can also be seen by noting that if $f(u)$ solves $(2.4)$ on $\left[u_{o}, \infty\right)$, then so does $f_{\delta} \equiv f(u+\delta)_{\mid\left[u_{o}, \infty\right)}$ for any $\delta \geqq 0$. Equation (2.8) shows that $f_{\delta}$ has the expansion

$$
\begin{aligned}
f_{\delta}=1 & +f_{1} e^{-v \delta} e^{-v u}+f_{2} e^{-2 v \delta} e^{-2 v u}+\cdots \\
& +f_{14} e^{-14 v \delta} e^{-14 v u}+f_{\log } e^{-15 v \delta} u e^{-15 v u}+\left(f_{15}+\delta f_{\log }\right) e^{-15 v \delta} e^{-15 v u}+\cdots,
\end{aligned}
$$

and uniqueness arguments yield the result.

Proposition 2.1. Let $P$ be the antipodal map of the sphere into itself and let $R_{\varphi}$ be the rotation of the sphere around the $z$-axis by an angle $\varphi$, suppose that

$$
f\left(u_{o}\right) \circ P=f\left(u_{o}\right) \circ R_{\varphi}=f\left(u_{o}\right)
$$

for $0 \leqq \varphi \leqq 2 \pi$. We have

$$
f_{\log } \not \equiv 0 \text {, }
$$

unless the function $f_{1}$ in the expansion (2.8) vanishes.

Proof. Since symmetries of the initial data which are also symmetries of $\dot{g}_{a b}$ are preserved by evolution via (2.4), it is not too difficult to show tha: all the expansion coefficients in (2.8) satisfy

$$
f_{i} \circ P=f_{i} \circ R_{\varphi}=f_{i} .
$$

It follows from Lemma 2.1 that

$$
f_{1}=\frac{2 a}{3} \times P_{2}(\cos \theta)
$$

where $a$ is a real constant and $P_{2}$ is a Legendre polynomial (we use the normalization of Legendre polynomials of Ref. [1]; we have found it convenient to introduce the factor $2 / 3$ in (2.18) to keep down the numerical value of some of the coefficients appearing in the functions $f_{i}$ for large $i$ ), and that $B_{\alpha}^{-}=0$, thus $f_{\log }$ is uniquely determined by $a$. We have written a REDUCE code which effectively implements the procedure described in Lemma 2.1 assuming invariance of $f\left(u_{o}\right)$ under rotations around the $z$ axis. The analysis is considerably simplified by noting that the coefficients $f_{i}$ must be linear combinations of spherical harmonics of order less than or equal to $2 i$ : this reduces the task of solving the equatiions $L_{i} f_{i}=g_{i}$ to algebraic operations in finite dimensional spaces. The change of variables $x=\cos \theta$ further simplifies the problem to manipulations with polynomials in $x$ of order $2 i \leqq 30$. To illustrate the results one obtains, here follow the first five functions $f_{i}$ :

$$
f_{1}=a \times\left(x^{2}-\frac{1}{3}\right),
$$




$$
\begin{aligned}
f_{2}= & -a^{2} \times\left(\frac{23}{78} x^{4}-\frac{47}{39} x^{2}+\frac{49}{234}\right) \\
f_{3}= & a^{3} \times\left(\frac{997}{5226} x^{6}-\frac{36697}{47034} x^{4}+\frac{25309}{15678} x^{2}-\frac{8899}{47034}\right), \\
f_{4}= & -a^{4} \times\left(\frac{4519475}{27990456} x^{8}-\frac{1636874143}{2078291358} x^{6}+\frac{73857848527}{45722409876} x^{4}\right. \\
& \left.-\frac{17112915619}{7620401646} x^{2}+\frac{18150013841}{91444819752}\right), \\
f_{5}= & a^{5} \times\left(\frac{646556531}{4114597032} x^{10}-\frac{5057087713397}{5567049784296} x^{8}+\frac{84695216485153}{38191860727020} x^{6}\right. \\
& \left.\quad-\frac{1554887482454485}{511770933742068} x^{4}+\frac{1086788290750781}{341180622494712} x^{2}-\frac{231262717823569}{1023541867484136}\right) .
\end{aligned}
$$

The length of the numerators and the denominators of the coefficients tends to grow rather rapidly with $i$, leading to rationals involving integers of more than 100 digits for $i \geqq 11$ (up to more than 210 digits for $i=15$ ), however with the normalization of $(2.18)$ the numerical values of the coefficients of the polynomials $a^{-i} f_{i}, 1 \leqq i \leqq 15$, are all of order $10^{-1}-10^{4}$. It takes about two and a half hours of CPU time on a VAX 8700 to obtain ${ }^{3}$

$$
\begin{aligned}
f_{\log } & \approx 1.009201657002 \times 10^{-10} \times a^{15} \times P_{4}(\cos \theta) \\
& \approx(0.2155750672866 a)^{15} \times P_{4}(\cos \theta) .
\end{aligned}
$$

This result has been obtained using integer arithmetic, so that the only error in the first equality in (2.19) is due to round-off when translating a rational into floating point notation: the exact value of $a^{-15} P_{4}(\cos \theta)^{-1} f_{\log }$ is a ratio of two integers of 109 and 118 digits which we can make available to anyone interested on request. In order to minimize the risk of programming errors we have built in several checks in the code to test the consistency of the results. Because we were quite perplexed by the numerical value of $a^{-15} P_{4}(\cos \theta)^{-1} f_{\log }$, which is at least 9 orders of magnitude smaller ${ }^{4}$ than the typical coefficients of the polynomials $a^{-i} f_{i}, 1 \leqq i \leqq 15$, we have written a MACSYMA code $^{5}$ which checked the REDUCE results by reading the output of the REDUCE calculation and verifying whether Eq. (2.4) was satisfied up to terms decaying faster than $e^{-15 v u}$.

\footnotetext{
${ }^{3}$ We had to make various optimizations to our code to be able to obtain (2.19) without exceeding the job limit of 4 hours of CPU time on the machine we were using. The same result (to the accuracy of (2.19)) can be obtained by running the code in E-30 floating point precision in about 15 minutes of CPU time

${ }^{4}$ Equation (2.19) clearly shows that a change of the normalization of $a$ by a factor $\approx 5$ would lead to a coefficient of $P_{4}$ in $f_{\text {log }}$ of order 1 - this will however not change the relative size of typical coefficients in $f_{\text {log }}$ as compared to typical coefficients in $f_{15}$.

5 The MACSYMA code was a "brute force one," without any fancy time- and memory-saving tricks; the checking run took about one and a half hours of CPU time on Sequent Symmetry. Both our codes together with all the coefficients $f_{i}$ up to $i=15$ are available on request.
} 
Equation (2.19) shows that $f_{\log }$ does not vanish unless $a=0$, which had to be established.

Lemma 2.1 and Proposition 2.1 imply that for "generic final states" of RT space-times the function $f_{\log }$ does not vanish:

Proposition 2.2. There exists an open dense subset $\Omega \subset \mathbb{R}^{12}$ such that iff is a solution of (2.4) for which $\left(B_{\alpha}^{+}, B_{\alpha}^{-}\right) \in \Omega,\left(B_{\alpha}^{+}, B_{\alpha}^{-}\right)$as in Lemma 2.1, then

$$
f_{\log } \neq 0 \text {. }
$$

Proof. As shown in Lemma $2.1, f_{\log }$ is determined uniquely by $\left(B_{\alpha}^{+}, B_{\alpha}^{-}\right)$. Consider the set $C \Omega$ of $\left(B_{\alpha}^{+}, B_{\alpha}^{-}\right)$for which $f_{\log } \equiv 0$. Since $f_{\log }$ is a polynomial in $\left(B_{\alpha}^{+}, B_{\alpha}^{-}\right), C \Omega$ is closed and thus the set $\Omega$ of $\left(B_{\alpha}^{+}, B_{\alpha}^{-}\right)$for which $f_{\log } \not \equiv 0$ is open. Suppose that $\Omega$ is not dense, therefore there exists a $p \in C \Omega$ and an open neighbourhood $\mathscr{U}$ of $p$ such that $\mathscr{U} \subset C \Omega$, therefore $f_{\log } \mid \mathscr{U}=0$. But a polynomial vanishing on an open set identically zero, which contradicts ${ }^{6}$ Proposition 2.1 , and proves our claim.

Let us show that the non-vanishing of $f_{\mathrm{log}}$ implies a form of singular behaviour of $\mathscr{H}^{+}$in the corresponding RT space-time:

Proposition 2.3. Suppose that the function $f_{\log }$ of the expansion (2.8) does not vanish. There exists no extensions of the corresponding RT space-time ${ }^{4} \mathscr{M}$ across $\mathscr{H}^{+}$, vacuum or otherwise, with a metric of $C^{123}$ differentiability class.

Remark. Let us mention that all scalar functions of the form

$$
C\left[\nabla^{\mu_{1}} \cdots \nabla^{\mu_{i}} R^{\alpha_{1} \beta_{1} \gamma_{1} \delta_{1}} \cdots \nabla_{v_{1}} \cdots \nabla_{v_{k}} R_{\alpha_{j} \beta_{j} \gamma_{j} \delta_{j}}\right]
$$

where $C[\cdots]$ denotes a (total) contraction operation over the indices, are uniformly bounded in a neighbourhood of $\mathscr{H}^{+}$in ${ }^{4} \mathscr{M}$, which follows immediately from the fact that in the coordinate system used in (2.3) $g_{\mu v}, g^{\mu v}$ and all partial derivatives thereof are uniformly bounded on $\mathcal{O}_{\varepsilon}=\left\{r \geqq \varepsilon, u \geqq u_{o}+\varepsilon\right\}$, for any $\varepsilon>0\left(u \geqq u_{o}\right.$ if $f\left(u_{o}\right)$ is smooth). The proof below shows that at least one entry of the tensor $\nabla_{v_{1}} \cdots \nabla_{v_{121}} R_{\alpha \beta \gamma \delta}$ will blow up at $\mathscr{H}^{+}$, whatever coordinate system one chooses, even though every scalar function constructed out of this tensor by contractions with products of $g^{\mu v}, g_{\rho \sigma}$ and $\nabla_{\mu_{1}} \cdots \nabla_{\mu_{j}} R_{\alpha_{k} \beta_{k} \gamma_{k} \delta_{k}}$ will be bounded on $\mathcal{O}_{\varepsilon}$.

Proof. Suppose that there exists an extension ${ }^{4} \tilde{\mathscr{M}}$ of ${ }^{4} \mathscr{M}$ with a metric of $C^{123}$ differentiability class. We then have $R^{\alpha \beta \gamma \delta} R_{\alpha \beta \gamma \delta} \in C^{121}\left({ }^{4} \tilde{M}\right)$, and a SHEEP calculations gives

$$
R^{\alpha \beta \gamma \delta} R_{\alpha \beta \gamma \delta}=48 m^{2} r^{-6}
$$

on ${ }^{4} \mathscr{M}$, therefore we can extend $r$ to a function $\tilde{r} \in C^{121}\left({ }^{4} \tilde{\mathscr{M}}\right)$ by setting

$$
\tilde{r}=\left\{R^{\alpha \beta \gamma \delta} R_{\alpha \beta \gamma \delta} /\left(48 m^{2}\right)\right\}^{-1 / 6} \phi,
$$

\footnotetext{
${ }^{6}$ To obtain further evidence that the polynomial $f_{\text {log }}\left(B_{\alpha}^{+}, B_{\alpha}^{-}\right)$does not vanish identically we have also analyzed, using our REDUCE code, the case of axially symmetric initial data without imposing the parity condition $f\left(u_{o}\right) \circ P=f\left(u_{o}\right)$. In such a case $f_{5}$ is determined by $f_{1}$ up to the addition of $b^{5} \times P_{3}(\cos \theta)$, where $b$ is a real constant, and using the same normalization for $f_{1}$ as in (2.18) the REDUCE code gives $f_{\log } \approx\left\{(0.2155750672866 a)^{15}-0.6581020070622 \times a^{5} b^{10}\right\} \times P_{4}(\cos \theta)$; this result has also been obtained using integer arithmetics, so that the $\approx$ accounts only for round-off error of the translation of a rational number into floating point notation (and subsequently taking the fifteenth root in the first factor)
} 
where $\phi \in C^{\infty}\left({ }^{4} \tilde{\mathscr{M}}\right)$ is equal to 1 in ${ }^{4} \mathscr{M}$ and in a neighborhood of $\mathscr{H}^{+}$, and $\phi=0$ in a neighborhood of the points for which $R^{\alpha \beta \gamma \delta} R_{\alpha \beta \gamma \delta}$ vanishes. According to SHEEP, on ${ }^{4} \mathscr{M}$ the scalar $\nabla^{\mu} R^{\alpha \beta \gamma \delta} \nabla_{\mu} R_{\alpha \beta \gamma \delta}$ takes the form

$$
\nabla^{\mu} R^{\alpha \beta \gamma \delta} \nabla_{\mu} R_{\alpha \beta \gamma \delta}=\frac{720}{r^{8}}\left(-\frac{R}{2}+2 r f^{-1} \frac{\partial f}{\partial u}+\frac{2 m}{r}\right) .
$$

Since by hypothesis $r$ can be extended to ${ }^{4} \tilde{\mathscr{M}}$ in a $C^{121}$ way and $\nabla^{\mu} R^{\alpha \beta \gamma \delta} \nabla_{\mu} R_{\alpha \beta \gamma \delta} \in$ $C^{120}\left({ }^{4} \tilde{\mathscr{M}}\right)$ it follows from (2.21) that the function

$$
\psi=-\frac{R}{2}+2 r f^{-1} \frac{\partial f}{\partial u}
$$

can be extended to ${ }^{4} \tilde{\mathscr{M}}$ as a $C^{120}\left({ }^{4} \tilde{\mathscr{M}}\right)$ function. Inserting the expansion $(2.8)$ in (2.22) one finds

$$
\psi=1+\psi_{1} e^{-v u}+\cdots+\psi_{\log } u e^{-15 v u}+\cdots,
$$

with

$$
\psi_{\log }=-102 f_{\mathrm{log}}
$$

at $r=2 m$, and the argument of the proof of Theorem 4.1 of [9] shows that there exists a geodesic $\Gamma$ in ${ }^{4} \tilde{\mathscr{M}}$ on which $\frac{d^{120} \psi}{d s^{120}}$ blows up as $\Gamma$ crosses $\mathscr{H}^{+}$, which contradicts $\psi \in C^{120}\left({ }^{4} \tilde{\mathscr{M}}\right)$ and proves our claim.

It follows from the results of this section that the potentially singular character of $\mathscr{H}^{+}$is controlled by the "asymptotic data" $\left(B_{\alpha}^{+}, B_{\alpha}^{-}\right) \in \mathbb{R}^{12}$. Obviously these "asymptotic data" do not determine the whole space-time, though it is tempting to conjecture that the collection of all $f_{i, 0}$ 's determines every RT space-time uniquely; we shall however not attempt to analyze that problem.

2.2. Other Topologies. Throughout this section we shall assume that $m<0$ and that the genus $g\left({ }^{2} \mathscr{M}\right)$ of ${ }^{2} \mathscr{M}$ satisfies $g\left({ }^{2} \mathscr{M}\right) \geqq 2$ (for the remaining cases, cf. e.g. [9]). By conformally rescaling the metric $\stackrel{\circ}{g}_{a b}$ and redefining $f\left(u_{o}\right)$ if necessary we can without loss of generality assume that $R\left(\dot{g}_{a b}\right)=-2$. Given a solution of (2.4) we can define

$$
A=\left\{a \in \mathbb{R}: \exists C \in \mathbb{R} \text { such that }\left|(f-1) \hat{u}^{-a}\right| \leqq C\right\},
$$

where

$$
\hat{u}=\exp \left\{-\frac{u}{4 m}\right\}
$$

set

$$
\hat{v}=\lim \sup A .
$$

From the existence of the expansion (2.6) it follows that if $\hat{v} \neq \infty$ then $\hat{v} \in A$, and if we set $\hat{v}_{i} \equiv 4|m| v_{i}, v_{i}$ as in (2.6), we also have

$$
\hat{v} \geqq \hat{v}_{1} \equiv \frac{\mu_{1}\left(\mu_{1}+2\right)}{3},
$$




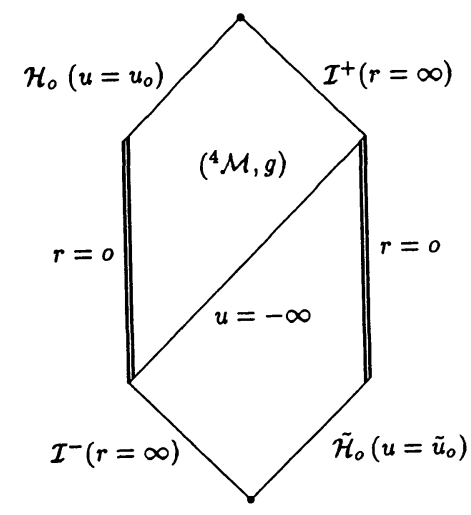

Fig. 2.2. Maximal vacuum RT extensions of $\left({ }^{4} \mathscr{M}, g\right), m<0, g\left({ }^{2} \mathscr{M}\right)>1$

where $\mu_{1}$ is the first non-trivial eigenvalue of $-\Delta_{o}$. For generic $f\left(u_{o}\right)$ one expects $\hat{v}=\hat{v}_{1}$ : this is indeed the case if $f\left(u_{o}\right)$ is generic and $\ln f\left(u_{o}\right)$ is "small enough," as follows from Theorem 3.2. On the other hand Corollary 3.1, point 2, implies that there exist (non-generic) $f\left(u_{o}\right)$ 's, not identically equal to 1 , for which $\hat{v} \geqq \frac{\mu_{2}\left(\mu_{2}+2\right)}{3}$, where $\mu_{2}$ is the second non-trivial eigenvalue of $-\Delta_{o}$ (an example in which $\hat{v}=\infty$ is given by $f=1$, which solves (2.4) and leads to the so-called "DS-metrics" [12]). If

$$
\hat{v} \geqq 2 \text {, }
$$

the corresponding space-time $\left({ }^{4} \mathscr{M}, g\right)$ can be extended across the boundary $u=-\infty$ $(\hat{u}=0)$ to a space-time $\left({ }^{4} \tilde{M}, \tilde{g}\right)$ with a continuous metric $\tilde{g}$ the degree of differentiability of which will depend upon $\hat{v}$ [9]. The global structure of $\left({ }^{4} \tilde{\mathscr{M}}, \tilde{g}\right)$ is displayed in Fig. 2.2. If $\hat{v}<2$ the "hypersurface" $u=-\infty$ is expected to be singular, although we have not been able to prove such a claim (cf. also the Remark following Proposition 2.3).

As is well known, metrics $\stackrel{\circ}{g}_{a b}$ satisfying $R\left(\stackrel{\circ}{g}_{a b}\right)=-2$ may be used to parametrize the Teichmüller space $\mathscr{T}\left({ }^{2} \mathscr{M}\right)$ [11], which allows one to consider $\mu_{1}$ as a function on $\mathscr{T}\left({ }^{2} \mathscr{M}\right)$. It is also known that $\mu_{1}$ varies continuously over $\mathscr{T}\left({ }^{2} \mathscr{M}\right)$, is uniformly bounded from above and tends to zero as one approaches the boundaries of $\mathscr{T}\left({ }^{2} \mathscr{M}\right)$. For $g\left({ }^{2} \mathscr{M}\right)=2$ it has been shown ${ }^{7}$ by Jenni [14] that there exists a metric on ${ }^{2} \mathscr{M}$ for which

$$
3.83<\mu_{1}<3.85
$$

and also that we have the bound

$$
\sup _{g\left({ }^{2} \mathscr{M}\right) \geqq 2, \dot{g}_{a b} \in \mathscr{T}\left({ }^{2} \mathscr{M}\right)} \mu_{1}<4.81
$$

7 The following has been explained to us by C. Hodgson: it follows easily from the Gauss-Bonnet theorem that the diameter of a genus $g$ manifold with a metric $\stackrel{\circ}{g}_{a b}$ for which $R\left(\dot{g}_{a b}\right)=-2$ tends to infinity as $g \rightarrow \infty$, which together with e.g. Theorem 8 of Ref. [4] shows that $\mu_{1} \leqq \hat{\mu}(g)$, with $\hat{\mu}(g) \searrow 1 / 4$ as $g \rightarrow \infty$. This implies that there exists $g_{o}$ such that if $g\left({ }^{2} \mathscr{M}\right) \geqq g_{o}$ then $\hat{v}_{1}<2$ for all metrics $\stackrel{\circ}{a b}_{a b}$ on ${ }^{2} \mathscr{M}$ such that $R\left(\dot{g}_{a b}\right)=-2$; thus for $g\left({ }^{2} \mathscr{M}\right) \geqq g_{o}$ the analysis that follows applies to non-generic $f\left(u_{o}\right)$ only. 
(it is actually expected that the supremum over $g$ is attained for $g=2$, and that for $g\left({ }^{2} \mathscr{M}\right)=2$ the supremum over $\mathscr{T}\left({ }^{2} \mathscr{M}\right)$ is attained by the metric considered by Jenni). For Jenni's metric one obtains

$$
5.44<\hat{v}-2<5.51
$$

which shows that at least for $g\left({ }^{2} \mathscr{M}\right)=2$ there will exist a large set of metrics for which $\hat{v}_{1}>2$. From the definition of $\hat{v}$ and from (2.6) if $\hat{v} \neq \infty$ we have

$$
f=1+f_{1} \hat{u}^{\hat{v}}+r_{1}, \quad f_{1} \neq 0 \text {, }
$$

with $r_{1}=O\left(\hat{u}^{\hat{v}+\epsilon}\right)$ for some $\varepsilon>0$. From Eq. (3.3) of [9] it follows that the metric on ${ }^{4} \mathscr{M}$ can be extended across the boundary $\hat{u}=0$ in a $C^{\operatorname{lnt}[\hat{v}-2]}$ way, where $\operatorname{Int}[x]$ stands for the integer part of $x$, but for $\hat{v} \notin \mathbb{N}$ the metric will not be $C^{\operatorname{Int}[\hat{v}-1]}$ in the coordinate system used ${ }^{8}$. Thus for generic pairs $\left(\stackrel{\circ}{g}_{a b}, f_{1}\right)$, with $\stackrel{8}{g}$ such that $\mu_{1}\left(\mu_{1}+2\right) \geqq 6$, the space-time metric will have some of its derivatives blowing up in the coordinate system used in [9]; for example Jenni's metric on ${ }^{2} \mathscr{M}$ will lead to $C^{5}$ but not $C^{6}$ extendible RT space-times in the coordinate system used in [9]. If (2.24) holds, an argument similar to the proof of Proposition 2.3 (note that both (2.20) and (2.21) hold irrrespective of the topology of ${ }^{2} \mathscr{M}$ ) shows that no $C^{\operatorname{Int}[\hat{v}+4]}$ extensions of a RT space-time exist when $\hat{v} \notin \mathbb{N}$ and the function $f_{1}$ in the expansion (2.27) does not vanish (by Theorem 3.2 this will be the case if e.g. $\ln f\left(u_{o}\right)$ is small enough and generic) - no details will be given.

\section{Generic "Final States" Versus Generic Initial Data}

The results presented in the previous sections show that RT space-times with "generic asymptotic data" in the sense of Proposition 2.2 are inextendible across $\mathscr{H}^{+}$in the class of manifolds with $C^{\infty}$ metrics. This leads immediately to the question, do generic Cauchy data for Eq. (2.4) lead to generic asymptotic parameters $\left(B_{\alpha}^{+}, B_{\alpha}^{-}\right)$? We believe that this is indeed the case, a partial answer to this question will be given in Theorem 3.2, Sect. 3.2 below. Before addressing this problem we shall need some results concerning the linearization of the RT equation, which are derived in the next section:

3.1. The Linearized Problem. Throughout this and the next section, the letter $C$ denotes a constant the value of which may vary from line to line; by $\stackrel{\circ}{a b}_{a b}$ we will denote a metric of constant scalar curvature $R_{o}=2$ for ${ }^{2} \mathscr{M}=S^{2}, R_{o}=0$ for ${ }^{2} \mathscr{M}=T^{2}$ and $R_{o}=-2$ otherwise. In the arguments that follow we shall assume that the reader is familiar with the methods and the results of [8] and [9], and we shall skip the non-essential details which may be filled in using either the results or methods of [8] and [9]. Let us simply recall here that from what has been proved in [8] it follows that for $f\left(u_{o}\right) \in H_{k}\left({ }^{2} \mathscr{M}\right), k \geqq 4$, there exists a solution of the RT equation satisfying $f \in C\left(\left[u_{o}, \infty\right), H_{k}\left({ }^{2} \mathscr{M}\right)\right) \cap C^{1}\left(\left[u_{o}, \infty\right), H_{k-4}\left({ }^{2} \mathscr{M}\right)\right) \cap C^{\infty}\left(\left(u_{o}, \infty\right) \times{ }^{2} \mathscr{M}\right)$.

\footnotetext{
${ }^{8}$ In the $S^{2}$ case a sufficient and necessary condition for a singular $\mathscr{H}^{+}$is the occurrence of $\log$ terms in the expansion (2.6), because for $\left(S^{2}, \stackrel{g}{g}_{a b}\right)$, with $\stackrel{\circ}{a b}_{a b}$ - the standard round metric, the spectrum of $-\Delta_{o}$ consists of integers; this will certainly not be the case for a generic $\left({ }^{2} \mathscr{M}, \stackrel{g}{g}_{a b}\right)$ with $R\left(\stackrel{\circ}{a b}_{a b}\right)=-2$. Whenever $\hat{v}=\mu_{1} \notin \mathbb{N}$ and $f_{1}$ in (2.27) does not vanish, the log terms become irrelevant
} 
Let $f_{t}$ be a one-parameter family of solutions of the modified RT equation (cf. $[9,20]$ for details), with $\left.f_{t}\right|_{t=0}=f,\left.\frac{d f_{t}}{d t}\right|_{t=0}=\varphi$ :

$$
\frac{\partial f_{t}}{\partial u}=-\frac{f_{t}^{3}}{24 m} \Delta_{o}\left[f_{t}^{2}\left(R_{o}+2 \Delta_{o} \ln f_{t}\right)\right]-f_{t} \sum_{i} \alpha_{i}\left(f_{t}\right) \phi_{i}-X^{a} \stackrel{\circ}{\nabla}_{a} f_{t},
$$

where

$$
\begin{aligned}
\alpha_{i}(f) & =c \oint \phi_{i} f d \mu_{o} \equiv \frac{c}{A} \int_{2 \mathscr{M}} \phi_{i} f d \mu_{o}, \\
A & =\int_{2 \cdot \mathscr{M}} d \mu_{o}, \\
X^{a} & =\sum_{i} \alpha_{i}(f) \stackrel{\circ}{\nabla}^{a} \phi_{i},
\end{aligned}
$$

with $c=0$ unless ${ }^{2} \mathscr{M}=S^{2}$; in this last case the $\phi_{i}$ 's form an $L^{2}$-orthonormal basis (with respect to $\oint$ ) of the space of $l=1$ spherical harmonics $\left(\Delta_{o} \phi_{i}=-2 \phi_{i}\right)$, and $c$ is a constant which we shall choose to satisfy

$$
c>\frac{\mu_{4}\left(\mu_{4}-R_{o}\right)}{12 m}
$$

(if ${ }^{2} \mathscr{M}=S^{2}$, then $\mu_{l}=l(l+1)$ ). We shall assume that the $f_{t}$ 's are normalized in such a way that

$$
\oint f_{t}^{-2}\left(u_{o}\right) d \mu_{o}=1 .
$$

It follows from (3.1) that $\varphi$ satisfies the equation

$$
\begin{aligned}
\frac{\partial \varphi}{\partial u}= & L \varphi+L_{1} \varphi \\
L \varphi= & -\frac{1}{12 m}\left[\Delta_{o}^{2}+2 \Delta_{o}\right] \varphi-\sum_{i} \alpha_{i}(\varphi) \phi_{i}, \\
L_{1} \varphi= & -\frac{f^{3}}{12 m} \Delta_{o}\left[f^{2} \Delta_{o}\left(\frac{\varphi}{f}\right)\right]-\frac{f^{3}}{12 m} \Delta_{o}\left(\frac{R \varphi}{f}\right)-\frac{\varphi f^{2}}{8 m} \Delta_{o} R \\
& -\sum_{i}\left\{\alpha_{i}(f)\left[\phi_{i} \varphi+\stackrel{\circ}{ }^{a} \phi_{i} \varphi_{, a}\right]+\alpha_{i}(\varphi)\left[\phi_{i} f+\stackrel{\circ}{\nabla}^{a} \phi_{i} f_{, a}\right]\right\}-L \varphi .
\end{aligned}
$$

If $f\left(u_{o}\right) \in H_{k}\left({ }^{2} \mathscr{M}\right), k \geqq 4$, it is simple to show by straightforward energy estimates that for $\varphi\left(u_{o}\right) \in H_{k}\left({ }^{2} \mathscr{M}\right)$ there exists a solution of (3.4) satisfying $\varphi \in C\left(\left[u_{o}, \infty\right)\right.$, $\left.H_{k-2}\left({ }^{2} \mathscr{M}\right)\right) \cap C^{\infty}\left(\left(u_{o}, \infty\right) \times{ }^{2} \mathscr{M}\right)$; using the methods of Appendix B of [8] one can then show that moreover $\varphi \in C\left(\left[u_{o}, \infty\right), H_{k}\left({ }^{2} \mathscr{M}\right)\right) \cap C^{1}\left(\left[u_{o}, \infty\right), H_{k-4}\left({ }^{2} \mathscr{M}\right)\right)$. Using the methods of [9] it can be shown that $\varphi$ has an expansion as in (2.6).

Define

$$
f^{o} \equiv P^{o} f \equiv \oint f d \mu_{o} \equiv \int_{2 \mathscr{M}} f d \mu_{o} / \int_{\mathscr{C}_{\mathscr{M}}} d \mu_{o} .
$$

1. If ${ }^{2} \mathscr{M}=S^{2}$, let $P: S^{2} \rightarrow S^{2}$ be the antipodal map, set

$$
f^{+} \equiv \frac{1}{2}(f \circ P+f) ; \quad f^{-} \equiv \frac{1}{2}(f \circ P-f),
$$


let $P^{+}$, respectively $P^{-}$, denote the $L^{2}$-orthogonal projection operator onto the second, respectively the third, non-trivial eigenspace, $\mathscr{E}^{+}$, respectively $\mathscr{E}^{-}$, of $-\Delta_{o}$. If $\left\{\phi_{\alpha}^{ \pm}\right\}$are $L^{2}$-orthonormal bases of $\mathscr{E}^{ \pm}$:

$$
\Delta_{o} \phi_{\alpha}^{ \pm}=-\mu^{ \pm} \phi_{\alpha}^{ \pm}
$$

set $\left(\phi_{\alpha}\right)=\left(\phi_{\alpha}^{+}, \phi_{\alpha}^{-}\right)$, and

$$
\bar{P}=P^{+}+P^{-}, \quad \tilde{P}=1-\bar{P}-P^{o} .
$$

2. If ${ }^{2} \mathscr{M} \neq S^{2}$, set $f^{+}=f, f^{-}=0$, let $P^{+}$denote the $L^{2}$-orthogonal projection operator onto the first non-trivial eigenspace, $\mathscr{E}^{+}$, of $-\Delta_{o}$, let $\left\{\phi_{\alpha}^{+}\right\}$be an $L^{2}$-orthonormal basis of $\mathscr{E}^{+}$:

$$
\Delta_{o} \phi_{\alpha}^{+}=-\mu^{+} \phi_{\alpha}^{+}, \quad \mu^{+}=\mu_{1}>0 ;
$$

we also define

$$
\bar{P} f=P^{+} f, \quad P^{-} f=0, \quad \tilde{P}=1-\bar{P}-P^{o}, \quad \mu^{-}=0,
$$

and $\left\{\phi_{\alpha}\right\}=\left\{\phi_{\alpha}^{+}\right\}, \quad\left\{\phi_{\alpha}^{-}\right\}=\{0\}$.

Whatever the topology of ${ }^{2} \mathscr{M}$, we thus have

$$
\begin{aligned}
P^{ \pm} \varphi & =\sum_{\alpha} A_{\alpha}^{ \pm} \phi_{\alpha}^{ \pm}, \quad A_{\alpha}^{ \pm}=\oint \phi_{\alpha}^{ \pm} \varphi d \mu_{o}, \\
\bar{P} \varphi & =\sum_{\alpha} A_{\alpha} \phi_{\alpha}, \quad\left(A_{\alpha}\right)=\left(A_{\alpha}^{+}, A_{\alpha}^{-}\right),
\end{aligned}
$$

and we define

$$
\begin{aligned}
(v=) v^{+} & \equiv \frac{1}{24 m} \mu^{+}\left(\mu^{+}-R_{o}\right), \\
v^{-} & \equiv \frac{1}{24 m} \mu^{-}\left(\mu^{-}-R_{o}\right) .
\end{aligned}
$$

Since the modified RT equation is area-preserving, ${ }^{9}$

$$
\oint f_{t}^{-2}(u) d \mu_{o}=1,
$$

it follows that for all $u \geqq u_{o}$ we have

$$
\oint f^{-3} \varphi d \mu_{o}=0 \Rightarrow \varphi^{o}=-\frac{1}{\oint f^{-3} d \mu_{o}} \oint f^{-3}(\bar{\varphi}+\tilde{\varphi}) d \mu_{o},
$$

where $\bar{\varphi}=\bar{P} \varphi$ and $\tilde{\varphi}=\tilde{P} \varphi$. From (3.4) and (3.11) one obtains

$$
\begin{aligned}
& \frac{d A_{\alpha}^{ \pm}}{d u}=-v^{ \pm} A_{\alpha}^{ \pm}+\sum_{\sigma= \pm, \beta} \Psi_{\alpha \beta}^{ \pm \sigma} A_{\beta}^{\sigma}+\Xi_{\alpha}^{ \pm}[\tilde{\varphi}], \\
& \Psi_{\alpha \beta}^{ \pm \pm}=\oint \phi_{\alpha}^{ \pm} L_{1} \phi_{\beta}^{ \pm} d \mu_{o}-\frac{1}{\oint f^{-3} d \mu_{o}} \oint L_{1}^{*} \phi_{\alpha}^{ \pm} d \mu_{o} \oint f^{-3} \phi_{\beta}^{ \pm} d \mu_{o},
\end{aligned}
$$

\footnotetext{
9 As pointed out in [20], this follows from the fact that the RT equation is area-preserving, and that the solutions of the modified RT equation differ from solutions of the RT equation only by a pull-back by a diffeomorphism
} 


$$
\Xi_{\alpha}^{ \pm}=\oint \tilde{\varphi} L_{1}^{*} \phi_{\alpha}^{ \pm} d \mu_{o}-\frac{1}{\oint f^{-3} d \mu_{o}} \oint L_{1}^{*} \phi_{\alpha}^{ \pm} d \mu_{o} \oint f^{-3} \tilde{\varphi} d \mu_{o},
$$

where $L_{1}^{*}$ is the formal adjoint of $L_{1}$, and

$$
\begin{gathered}
\frac{\partial \tilde{\varphi}}{\partial u}=L \tilde{\varphi}+\tilde{L}_{1} \tilde{\varphi}+\tilde{\Xi}\left[A_{\alpha}^{ \pm}\right], \\
\tilde{L}_{1} \tilde{\varphi}=L_{1} \tilde{\varphi}-\oint \tilde{\varphi} L_{1}^{*} 1 d \mu_{o}-\sum_{\alpha} \Xi_{\alpha}[\tilde{\varphi}] \phi_{\alpha}+\Gamma(\tilde{\varphi}), \\
\tilde{\Xi}\left[A_{\alpha}^{ \pm}\right]=\sum_{\alpha}\left(L_{1} \phi_{\alpha}-\oint L_{1} \phi_{\alpha} d \mu_{o}-\sum_{\beta} \Psi_{\beta \alpha} \phi_{\beta}+\Gamma\left(\phi_{\alpha}\right)\right) A_{\alpha},
\end{gathered}
$$

where

$$
\Gamma(\theta)=\frac{\oint f^{-3} \theta d \mu_{o}}{\oint f^{-3} d \mu_{o}}\left(\oint L_{1} 1 d \mu_{o}-L_{1} 1\right) .
$$

The sums in (3.16) and (3.17) (and in the matrix equations below) are implicitly over both the index and their "associated \pm ." We shall be interested in solutions of (3.4) for which

$$
A_{\alpha}^{ \pm}=e^{-v \pm u}\left(\widetilde{B}_{\alpha}^{ \pm}+F_{\alpha}^{ \pm}\right),
$$

where $\tilde{B}_{\alpha}^{ \pm}$are prescribed constants, and $F_{\alpha}^{ \pm} \stackrel{\mu \rightarrow \infty}{\longrightarrow} 0$. In order to prove existence of such solutions it turns out to be necessary to keep track separately of the even and odd parity parts ${ }^{10}, \tilde{\varphi}^{ \pm}$, of $\tilde{\varphi}$; if we set

$$
\zeta^{ \pm}=e^{v \pm u} \tilde{\varphi}^{ \pm}
$$

one finally obtains the following system of equations:

$$
\begin{aligned}
\frac{d F}{d u} & =\Psi(F+\tilde{B})+\xi\left[\zeta^{+}, \zeta^{-}\right] \\
\frac{\partial \zeta^{ \pm}}{\partial u} & =\left(L+v^{ \pm}\right) \zeta^{ \pm}+\sum_{\sigma= \pm} L^{ \pm \sigma} \zeta^{\sigma}+\rho^{ \pm}[F+\tilde{B}],
\end{aligned}
$$

with

$$
\begin{aligned}
F & =\left(\begin{array}{c}
F_{\alpha}^{+} \\
F_{\alpha}^{-}
\end{array}\right), \quad \tilde{B}=\left(\begin{array}{c}
\tilde{B}_{\alpha}^{+} \\
\tilde{B}_{\alpha}^{-}
\end{array}\right), \\
\Psi & =\left(\begin{array}{cc}
\Psi_{\alpha \beta}^{++} & e^{\left(v^{+}-v^{-}\right) u} \Psi_{\alpha \beta}^{+-} \\
e^{\left(v^{-}-v^{+}\right) u} \Psi_{\alpha \beta}^{-+} & \Psi_{\alpha \beta}^{--}
\end{array}\right), \\
\xi\left[\zeta^{+}, \zeta^{-}\right] & =\left(\begin{array}{c}
e^{v^{+} u} \Xi_{\alpha}^{+}\left[e^{-v^{+} u \zeta^{+}}+e^{-v^{-} u \zeta^{-}}\right] \\
e^{v^{-} u} \Xi_{\alpha}^{-}\left[e^{-v^{+} u \zeta^{+}}+e^{-v^{-} u \zeta^{-}}\right]
\end{array}\right), \\
\sum_{\sigma= \pm} L^{ \pm \sigma} \zeta^{\sigma} & \equiv e^{v^{ \pm} u}\left[\tilde{L}_{1}\left(e^{-v^{+} u \zeta^{+}}+e^{\left.-v^{-} u \zeta^{-}\right)}\right]^{ \pm},\right. \\
\rho^{ \pm}[F+\tilde{B}] & \equiv e^{v^{ \pm} u}\left[\tilde{\Xi}\left[e^{-v^{ \pm} u}\left(F_{\alpha}^{ \pm}+\tilde{B}_{\alpha}^{ \pm}\right)\right]\right]^{ \pm},
\end{aligned}
$$

${ }^{10}$ The idea of separating the even parity terms from the odd parity terms in the RT equation has also been considered by Rendall [16] 
$\Psi_{\alpha \beta}^{ \pm \pm}, \Xi_{\alpha}^{ \pm}, \tilde{L}_{1}$ and $\tilde{\Xi}$ being as in (3.13), (3.14), (3.16) and (3.17) respectively. With the choice of $c$ given by (3.3), it follows from the results of [9] by the same arguments as in the proof of Lemma 2.1 that any solution of the modified RT equation (3.1) on $S^{2}$ has the asymptotic expansion

$$
f=1+f_{1}^{+} e^{-v u}+\cdots+\left(f_{5}^{+}+f_{5}^{-}\right) e^{-v^{-} u}+O\left(e^{-6 v u}\right),
$$

with $f_{1}^{+} \in \mathscr{E}^{+}, f_{5}^{-} \in \mathscr{E}^{-}$,

$$
f_{1}^{+}=\sum \widetilde{B}_{\alpha}^{+} \phi_{\alpha}^{+}, \quad f_{5}^{-}=\sum \widetilde{B}_{\alpha}^{-} \phi_{\alpha}^{-}, \quad \Delta_{o} \phi_{\alpha}^{ \pm}=-\mu^{ \pm} \phi_{\alpha}^{ \pm} .
$$

Moreover, there exists a constant $C_{f}$ depending only upon $\left\|\ln f\left(u_{o}\right)\right\|_{H_{6}(2 \mathscr{M})}$ such that

$$
\begin{aligned}
& \|\ln f\|_{\left.C^{4(2} \mathscr{M}\right)}+e^{v^{+} u}\left\|f^{+}-1\right\|_{C^{4}(2 \mathscr{M})}+e^{v^{-} u}\left\|f^{-}\right\|_{C^{4}\left(\mathcal{2}^{2} \mathscr{M}\right)} \\
& \quad+e^{\left(v^{+}+v^{-}\right) u}\left\|f^{-}-f_{5}^{-} e^{-v^{-} u}\right\|_{C^{4}\left(\mathcal{C}^{2} \mathscr{M}\right)} \leqq C_{f}
\end{aligned}
$$

and it follows from Proposition 3.1, point 1, and the results of [8], that for any $\varepsilon>0$ there exists a $\delta>0$ such that if $\left\|\ln f\left(u_{o}\right)\right\|_{H_{6}{ }^{2}, \mathscr{M}} \leqq \delta \Rightarrow C_{f} \leqq \varepsilon$. A straightforward analysis, which requires somewhat tedious parity considerations if ${ }^{2} \mathscr{M}=S^{2}$, shows the following key lemma:

Lemma 3.1. Let $C_{f}$ be the constant defined by (3.25). There exists a constant $C(K)$ such that if $C_{f} \leqq K$, then

$$
\begin{aligned}
\left|\alpha_{i}(f)\right| & \leqq C C_{f} e^{-\left(v^{+}+v^{-}\right) u}, \\
|\Psi| & \leqq C C_{f} e^{-v^{+} u}, \\
|\xi| & \leqq C C_{f} e^{-v^{+} u}\left(\left\|\zeta^{+}\right\|_{L^{1}\left(2^{2} \mathscr{M}\right)}+\left\|\zeta^{-}\right\|_{L^{1}\left(2^{2} \mathscr{M}\right)}\right), \\
\left|\rho^{ \pm}(X)\right| & \leqq C C_{f} e^{-v^{+} u}\|X\|_{\mathbb{R}^{12}},
\end{aligned}
$$

and the operators $L^{ \pm \pm}$can be written in the form

$$
\sum_{i=0}^{4} A_{\alpha_{1} \cdots \alpha_{i}}^{ \pm \pm} \stackrel{\circ}{\nabla}^{\alpha_{1}} \cdots \stackrel{\circ}{\alpha_{\alpha_{i}}}
$$

for some tensors $A_{\alpha_{1} \cdots \alpha_{i}}^{ \pm \pm}$satisfying

$$
\left|A_{\alpha_{1} \cdots \alpha_{i}}^{ \pm \pm}\right| \leqq C C_{f} e^{-v^{+} u}
$$

$A_{\alpha_{1} \cdots \alpha_{i}}^{ \pm \pm}$smooth for all $u>u_{o}$ and uniformly $C^{2}$ for $u \geqq u_{o}$.

Let us start by analysing Eq. (3.20) assuming that $\xi$ is a given function of $(u, p) \in\left[u_{o}, \infty\right) \times{ }^{2} \mathscr{M}$ :

Lemma 3.2. For $\sigma>0$ and $k, m \in \mathbb{N}$, let $X_{k}^{\sigma}\left(\mathbb{R}^{m}\right)=\left\{F=e^{-\sigma u} G: G \in C^{k}\left(\left[u_{o}, \infty\right), \mathbb{R}^{m}\right)\right\}$, define

$$
\|F\|_{X_{k}^{o}}=\left\|e^{\sigma u} F\right\|_{C^{k}\left(\left[u_{o}, \infty\right), \mathbb{R}^{m}\right)},
$$

suppose that $\mathscr{L}: X_{k+1}^{\sigma} \rightarrow X_{k}^{\sigma}$ is defined by

$$
\begin{gathered}
\mathscr{L} F=\frac{d F}{d u}-\Psi F, \\
\Psi \in X_{k}^{v}\left(G L\left(\mathbb{R}^{m}, \mathbb{R}^{m}\right)\right), \quad v>0 .
\end{gathered}
$$


Then $\mathscr{L}$ is an isomorphism, in particular there exists a constant $C$ such that for every $\xi \in X_{k}^{\sigma}$, there exists a unique solution of the equation

$$
\frac{d F}{d u}=\Psi F+\xi
$$

satisfying

$$
\|F\|_{X_{k+1}^{\sigma}} \leqq C\|\xi\|_{X_{k}^{\sigma}}
$$

Proof. 1. Surjectivity: it is sufficient to show surjectivity on $\left[u_{1}, \infty\right)$, with some $u_{1}$ large enough, since any solution defined on $\left[u_{1}, \infty\right)$ can be uniquely continued backwards in $u$ by standard theorems on solutions of linear ODE's on compact intervals. Consider the problem

$$
\frac{d F_{i+1}}{d u}=\Psi F_{i}+\xi
$$

$F_{0}=0$, thus

$$
F_{i+1}=-\int_{u}^{\infty}\left(\Psi F_{i}+\xi\right)(s) d s
$$

and

$$
\begin{aligned}
\left|\left(F_{i+1}-F_{i}\right)(u)\right| & \leqq \int_{u}^{\infty}\left|\Psi\left(F_{i}-F_{i-1}\right)(s)\right| d s \\
& \leqq \frac{e^{-(\sigma+v) u}}{\sigma+v}\|\Psi\|_{X_{o}^{v}}\left\|F_{i}-F_{i-1}\right\|_{X_{0}^{\sigma}},
\end{aligned}
$$

so that for $u \in\left[u_{1}, \infty\right)$ we get

$$
\left\|F_{i+1}-F_{i}\right\|_{X_{0}^{\sigma}} \leqq \frac{e^{-v u_{1}}}{\sigma+v}\|\Psi\|_{X_{o}^{v}}\left\|F_{i}-F_{i-1}\right\|_{X_{0}^{\sigma}}
$$

and if $u_{i}>\frac{1}{v} \ln \left(\frac{\|\Psi\|_{X_{o}^{v}}}{\sigma+v}\right)$ the contraction mapping principle shows the existence of a fixed point $F$ for the problem (3.37), which solves (3.34) and is in $X_{k+1}^{\sigma}$.

2. Injectivity: Let $F_{1}, F_{2}$ satisfy (3.34), then we have

$$
\frac{d\left(F_{1}-F_{2}\right)}{d u}=\Psi\left(F_{1}-F_{2}\right), \quad F_{1}-F_{2} \stackrel{u \rightarrow \infty}{\longrightarrow} 0 .
$$

Suppose that $\left\|F_{1}-F_{2}\right\|_{\mathbb{R}^{m}}>0$, then $\ln \left\|F_{1}-F_{2}\right\|_{\mathbb{R}^{m}}$ is differentiable and from (3.38) one obtains

$$
\begin{aligned}
& \frac{d\left(\ln \left\|F_{1}-F_{2}\right\|_{\mathbb{R}^{m}}\right)}{d u} \geqq-\|\Psi\|_{X_{o}^{v}} e^{-v u} \\
& \quad \Rightarrow\left\|\left(F_{1}-F_{2}\right)\left(u_{1}\right)\right\|_{\mathbb{R}^{m}} \leqq \exp \left\{\frac{\|\Psi\|_{X_{o}^{v}} e^{-v u_{1}}}{v}\right\}\left\|\left(F_{1}-F_{2}\right)\left(u_{2}\right)\right\|_{\mathbb{R}^{m}} \text { for } \quad u_{2} \geqq u_{1} .
\end{aligned}
$$

Letting $u_{2} \rightarrow \infty$ one obtains $\left\|\left(F_{1}-F_{2}\right)\left(u_{1}\right)\right\|=0$ which contradicts the assumption 
that $\left\|F_{1}-F_{2}\right\|_{\mathbb{R}^{m}}>0$, i.e., there exists $\tilde{u}$ such that $\left(F_{1}-F_{2}\right)(\tilde{u})=0$. In this case, $F_{1} \equiv F_{2}$ follows from standard results for first order ODE's.

3. To prove (3.35), note that $\mathscr{L}$ is a bounded linear bijection from $X_{k+1}^{\sigma}$ to $X_{k}^{\sigma}$ thus, by the open mapping theorem, $\mathscr{L}^{-1}$ is a continuous linear operator from $X_{k}^{\sigma}$ to $X_{k+1}^{\sigma}$, which implies (3.35).

Lemma 3.2 shows that Eq. (3.20) can be solved for $F$ in terms of $\tilde{B}$ and $\zeta^{ \pm}$. By well known results, there exists functions $R_{\alpha \beta}^{ \pm \pm}$such that

$$
F_{\alpha}^{ \pm}=\int_{u}^{\infty} \sum_{\sigma= \pm, \beta} R_{\alpha \beta}^{ \pm \sigma}(u, s)\left\{\xi_{\beta}^{\sigma}\left[\zeta^{ \pm}(s)\right]+\sum_{\rho= \pm, \gamma} \Psi_{\beta \gamma}^{\sigma \rho} \tilde{B}_{\gamma}^{\rho}\right\} d s .
$$

Inserting (3.39) into (3.21), one obtains an equation of the form

$$
\begin{aligned}
\frac{\partial \zeta^{ \pm}}{\partial u}(u, x)= & {\left[\left(L+v^{ \pm}\right) \zeta^{ \pm}+\sum_{\sigma= \pm} L^{ \pm \sigma} \zeta^{\sigma}+\tilde{\rho}^{ \pm}(\tilde{B})\right](u, x) } \\
& +\int_{u}^{\infty} d s \int_{2 \mathscr{M}_{\sigma}= \pm} \sum^{ \pm \sigma}\left(u, s, x, x^{\prime}\right) \xi^{\sigma}\left(s, x^{\prime}\right) d \mu_{o}\left(x^{\prime}\right),
\end{aligned}
$$

which has the amusing property that the derivative $\frac{\partial \zeta^{ \pm}}{\partial u}$ at time $u$ depends on $\xi^{ \pm}(v)$ for all $v \geqq u$. To avoid the supplementary step of proving estimates on $R^{ \pm \pm}$, rather than analysing (3.40) we shall consider the system (3.20), (3.21) directly. We shall need the following Lemma, which gives information about solutions of Eq. (3.21) when $\rho^{ \pm}$are considered as being given functions of $(u, p)$ :

Lemma 3.3. Let $\zeta=\left(\zeta^{+}, \zeta^{-}\right), \rho=\left(\rho^{+}, \rho^{-}\right)$, set

$$
\begin{aligned}
& \|\zeta\|_{H_{k}\left({ }^{2} \mathscr{M}\right)}^{2}=\left\|\zeta^{+}\right\|_{H_{k}\left({ }^{2} \mathscr{M}\right)}^{2}+\left\|\zeta^{-}\right\|_{H_{k}\left({ }^{2} \mathscr{M}\right)}^{2}, \\
& \|\rho\|_{H_{k}\left({ }^{2} \mathscr{M}\right)}^{2}=\left\|\rho^{+}\right\|_{H_{k}\left(^{2} \mathscr{M}\right)}^{2}+\left\|\rho^{-}\right\|_{H_{k}\left(^{2} \mathscr{M}\right)}^{2} .
\end{aligned}
$$

There exists constants $C_{1}, C_{2}$ such that for all $u_{1}$ satisfying

$$
u_{1} \geqq \stackrel{\circ}{1}_{1}=\frac{1}{v} \ln \left(C_{1} C_{f}\right),
$$

where $C_{f}$ has been defined in (3.25), all solutions $\zeta^{ \pm} \in C^{1}\left(\left[u_{1}, \infty\right), L^{2}\left({ }^{2} \mathscr{M}\right)\right) \cap$ $C\left(\left[u_{1}, \infty\right), H_{4}\left({ }^{2} \mathscr{M}\right)\right)$ of (3.21) satisfy, for $u \geqq u_{1}$,

$$
\|\zeta(u)\|_{L^{2}(2 \mathscr{M})}^{2} \leqq\left\|\zeta\left(u_{1}\right)\right\|_{L^{2}(2 \mathscr{M})}^{2}+C_{2} e^{-2 v u} \int_{u_{1}}^{u} e^{2 v s}\|\rho(s)\|_{L^{2}\left({ }^{2} \mathscr{M}\right)}^{2} d s .
$$

Proof. In what follows we shall assume ${ }^{2} \mathscr{M}=S^{2}$; for other topologies the proof is obtained by similar arguments. By expanding $\tilde{\varphi}^{ \pm}$in spherical harmonics and using straightforward approximation arguments (cf. e.g. [9] [Lemma 4.1]), one obtains

$$
\begin{aligned}
& \oint \zeta^{+} L \zeta^{+} d \mu_{o} \leqq-15 v \oint\left(\zeta^{+}\right)^{2} d \mu_{o}, \\
& \oint \zeta^{-} L \zeta^{-} d \mu_{o} \leqq-35 v \oint\left(\zeta^{-}\right)^{2} d \mu_{o},
\end{aligned}
$$

and similarly one proves that there exists a constant $C_{o}$ such that

$$
\oint \varphi d \mu_{o}=0 \Rightarrow \oint \varphi L \varphi d \mu_{o} \leqq-C_{o}\|\varphi\|_{H_{2}\left({ }^{2} \mathscr{M}\right)}^{2} .
$$


Set

$$
E(u)=\oint\left[\left(\zeta^{+}\right)^{2}+\left(\zeta^{-}\right)^{2}\right] d \mu_{o},
$$

let $\alpha, \beta>0, \alpha+\beta=1$; we have, by (3.44)-(3.46),

$$
\begin{aligned}
\frac{d E}{d u}= & 2 \oint \zeta^{+}(L+v) \zeta^{+} d \mu_{o}+2 \oint \zeta^{-}\left(L+v^{-}\right) \zeta^{-} d \mu_{o} \\
& +2 \sum_{\rho= \pm, \sigma= \pm} \oint \zeta^{\rho} L^{\rho \sigma} \zeta^{\sigma} d \mu_{o}+2 \sum_{ \pm} \oint \zeta^{ \pm} \rho^{ \pm} d \mu_{o} \\
\leqq & -(30 \alpha-2) v \oint\left(\zeta^{+}\right)^{2} d \mu_{o}-(70 \alpha-10) v \oint\left(\zeta^{-}\right)^{2} d \mu_{0} \\
& -2 \beta C_{o}\left(\left\|\zeta^{+}\right\|_{H_{2}\left({ }^{2} \mathscr{M}\right)}^{2}+\left\|\zeta^{-}\right\|_{H_{2}\left({ }^{2} \mathscr{M}\right)}^{2}\right) \\
& +2 \sum_{\rho= \pm, \sigma= \pm} \oint \zeta^{\rho} L^{\rho \sigma} \zeta^{\sigma} d \mu_{0}+\varepsilon E(u)+\frac{1}{\varepsilon} \sum_{ \pm}\left\|\rho^{ \pm}\right\|_{L^{2}(2 \mathscr{M})}^{2},
\end{aligned}
$$

where we have used $2 a b \leqq \frac{1}{\varepsilon} a^{2}+\varepsilon b^{2}$. Some integration by parts in the terms containing $\zeta^{ \pm} L^{ \pm \pm} \zeta^{ \pm}$gives

$$
\sum_{\rho= \pm, \sigma= \pm} \oint \zeta^{\rho} L^{\rho \sigma} \zeta^{\sigma} d \mu_{o} \leqq C C_{f} e^{-v u}\left(\left\|\zeta^{+}\right\|_{H_{2}\left({ }^{2} \mathscr{M}\right)}^{2}+\left\|\zeta^{-}\right\|_{H_{2}\left({ }^{2} \mathscr{M}\right)}^{2}\right)
$$

(cf. (3.30), (3.31)), so that with (3.41) and (3.42), one finally obtains

$$
\frac{d E}{d u} \leqq-(30 \alpha v-10 v-\varepsilon) E(u)-\left(2 C_{o} \beta-C C_{f} e^{-v u}\right)\|\zeta\|_{H_{2}\left({ }^{2} \mathscr{M}\right)}^{2}+\frac{1}{\varepsilon}\|\rho\|_{H_{2}\left(^{2} \mathscr{M}\right)}^{2} .
$$

Choosing

$$
\alpha=\beta=\frac{1}{2}, \quad \varepsilon=3 v, \quad e^{-v \dot{\mathrm{u}}_{1}}=\frac{C_{o}}{C C_{f}},
$$

one gets

$$
u \geqq u_{1} \geqq{\stackrel{u_{1}}{1}} \Rightarrow E(u) \leqq e^{2 v\left(u_{1}-u\right)} E\left(u_{1}\right)+C_{2} \int_{u_{1}}^{u} e^{2 v(s-u)}\|\rho(s)\|_{H_{2}\left({ }^{2} \mathscr{M}\right)}^{2} d s,
$$

for some constant $C_{2}$.

Theorem 3.1. Let $f \in C^{0}\left(\left[u_{0}, \infty\right), H_{6}\left({ }^{2} \mathscr{M}\right)\right) \cap C^{1}\left(\left[u_{o}, \infty\right), H_{2}\left({ }^{2} \mathscr{M}\right)\right)$ be a solution of the modified RT equation. There exists $\stackrel{\leftrightarrow}{1}_{1} \geqq u_{o}$ depending only upon $\left\|\ln f\left(u_{o}\right)\right\|_{\left.H_{6}{ }^{2} \mathscr{M}\right)}$ such that for all $u_{1} \geqq{\stackrel{u_{1}}{1}}_{1}, \tilde{B}_{\alpha}^{ \pm} \in \mathbb{R}^{12}$ and $\tilde{\varphi}_{0} \in \tilde{P} H_{4}\left({ }^{2} \mathscr{M}\right)$ there exists $\bar{\varphi}_{0} \in \bar{P} H_{4}\left({ }^{2} \mathscr{M}\right) \equiv$ $\left(1-\widetilde{P}-P^{o}\right) H_{4}\left({ }^{2} \mathscr{M}\right)$ with the property that the unique solution of (3.4) satisfying

$$
(\tilde{P} \varphi)\left(u_{1}\right)=\tilde{\varphi}_{0}, \quad \bar{P} \varphi\left(u_{1}\right)=\bar{\varphi}_{0},
$$

with $\varphi^{o}\left(u_{1}\right)=\left(P^{o} \varphi\right)\left(u_{1}\right)$ given by (3.11), satisfies, for $u \rightarrow \infty$,

$$
\begin{aligned}
\varphi^{ \pm} & =\sum_{\alpha} \tilde{B}_{\alpha}^{ \pm} \phi_{\alpha}^{ \pm} e^{-v \pm u}+O\left(e^{-\left(v+v^{ \pm}\right) u}\right), \\
\Delta_{o} \phi_{\alpha}^{+} & =-6 \phi_{\alpha}^{+}, \quad \Delta_{o} \phi_{\alpha}^{-}=-12 \phi_{\alpha}^{-} .
\end{aligned}
$$

Moreover there exists $\varepsilon_{0}>0$ such that if $\left\|\ln f\left(u_{o}\right)\right\|_{H_{6}\left({ }^{2} \mathscr{M}\right)} \leqq \varepsilon_{0}$, then $\dot{u}_{1}=u_{o}$. 
Proof. Set $F_{0}=0$, and for $i \geqq 1$ let $F_{i}, \zeta_{i}^{ \pm}$be solutions of the equations

$$
\begin{aligned}
\frac{d F_{i}}{d u} & =\Psi\left(F_{i}+\tilde{B}\right)+\xi\left[\zeta_{i}^{+}, \zeta_{i}^{-}\right], \\
\frac{\partial \zeta_{i+1}^{ \pm}}{\partial u} & =\left(L+v^{ \pm}\right) \zeta_{i+1}^{ \pm}+\sum_{\sigma= \pm} L^{ \pm \sigma} \zeta_{i+1}^{\sigma}+\rho^{ \pm}\left[F_{i}+\tilde{B}\right],
\end{aligned}
$$

with $\zeta_{i+1}^{ \pm}\left(u_{1}\right)=\zeta^{o \pm}\left(u_{1}\right)$. For $i \geqq 1$, we have

$$
\begin{aligned}
& \frac{d\left(F_{i+1}-F_{i}\right)}{d u}=\Psi\left(F_{i+1}-F_{i}\right)+\xi\left[\zeta_{i+1}^{+}-\zeta_{i}^{+}, \zeta_{i+1}^{-}-\zeta_{i}^{-}\right] \\
& \frac{\partial\left(\zeta_{i+1}^{ \pm}-\zeta_{i}^{ \pm}\right)}{\partial u}=\left(L+v^{ \pm}\right)\left(\zeta_{i+1}^{ \pm}-\zeta_{i}^{ \pm}\right)+\sum_{\sigma= \pm} L^{\sigma \pm}\left(\zeta_{i+1}^{\sigma}-\zeta_{i}^{\sigma}\right)+\rho^{ \pm}\left[F_{i}-F_{i-1}\right]
\end{aligned}
$$

with

$$
\left.\left(\zeta_{i+1}^{ \pm}-\zeta_{i}^{ \pm}\right)\left(u_{1}\right)=0 \quad\left(\Rightarrow \| \zeta_{i+1}^{ \pm}-\zeta_{i}^{ \pm}\right)\left(u_{1}\right) \|_{L^{2}(2 \mathscr{M})}=0\right),
$$

and Lemma 3.2 with $\sigma=v$ and (3.28) gives, for $u \geqq u_{1}$,

$$
\begin{aligned}
\left\|\left(F_{i+1}-F_{i}\right)(u)\right\|_{\mathbb{R}^{12}} & \leqq C e^{-v u} \sup _{s \geqq u_{1}} e^{v s}\left\|\xi\left[\zeta_{i+1}^{+}-\zeta_{i}^{+}, \zeta_{i+1}^{-}-\zeta_{i}^{-}\right](s)\right\|_{\mathbb{R}^{12}} \\
& \leqq C C_{f} e^{-v u} \sup _{s \geqq u_{1}} \sum_{ \pm}\left\|\left(\zeta_{i+1}^{+}-\zeta_{i}^{ \pm}\right)(s)\right\|_{L^{2}(2 \mathscr{M})} .
\end{aligned}
$$

This together with (3.29), (3.49), (3.50) and Lemma 3.3 implies, for $u \geqq u_{1}, u_{1}$ large enough,

$$
\left[\sum_{ \pm}\left\|\left(\zeta_{i+2}^{ \pm}-\zeta_{i+1}^{ \pm}\right)(u)\right\|_{L^{2}(2 \mathscr{M})}\right]^{2} \leqq C C_{f}^{4} e^{-2 v\left(u_{1}+u\right)}\left[\sup _{s \geqq u_{1}} \sum_{ \pm}\left\|\left(\zeta_{i+1}^{ \pm}-\zeta_{i}^{ \pm}\right)(s)\right\|_{L^{2}\left({ }^{2} \mathscr{M}\right)}\right]^{2}
$$

so that if

$$
C^{1 / 4} C_{f} e^{-v u_{1}}<1
$$

the sequence $\zeta_{i}{ }^{ \pm}(u)$ converges in $L^{2}\left({ }^{2} \mathscr{M}\right)$ for each $u$ to a function $\zeta_{\infty}^{ \pm}(u)$. It is straightforward to show that in fact $\zeta_{\infty}^{ \pm}(u) \in C\left(\left[u_{1}, \infty\right), H_{4}\left({ }^{2} \mathscr{M}\right)\right) \cap C^{\infty}\left(\left(u_{1}, \infty\right) \times{ }^{2} \mathscr{M}\right)$, and the remaining claims follow by methods similar to those of $[8,9]$. Note that it follows from the methods of $[8,9]$ that the constant $C_{f}$ in (3.25) depends only upon $\left\|\ln f\left(u_{o}\right)\right\|_{H_{6}\left({ }^{2} \mathscr{M}\right)}$, and in fact for $\left\|\ln f\left(u_{o}\right)\right\|_{H_{6}\left({ }^{2} \mathscr{M}\right)} \leqq 1$ it follows from Proposition 3.1, point 2 , that we have

$$
C_{f} \leqq C\left\|\ln f\left(u_{o}\right)\right\|_{H_{6}\left({ }^{2} \mathscr{M}\right)},
$$

which shows that for $\left\|\ln f\left(u_{o}\right)\right\|_{H_{6}\left({ }^{2} \mathscr{M}\right)}$ small enough we can set $u_{1}=u_{o}$.

3.2. The Nonlinear Equation. In this section we shall show how the genericity problem can be reduced to the linearised problem analysed in Sect. 3.1. Let us note the following:

Proposition 3.1. Consider the map $\mathscr{B}_{u_{o}, k}: H_{k}\left({ }^{2} \mathscr{M}\right) \rightarrow \mathbb{R}^{N}$ (respectively $\overline{\mathscr{B}}_{u_{o}, k}: H_{k}\left(S^{2}\right) \rightarrow$ $\left.\mathbb{R}^{N}\right)\left(N=12\right.$ if $\left.{ }^{2} \mathscr{M}=S^{2}\right)$ which to an initial datum $f\left(u_{o}\right) \in H_{k}\left({ }^{2} \mathscr{M}\right)$ assigns 
1. the coefficients $\left(B_{\alpha}^{ \pm}\right)$of the expansion (2.8) if ${ }^{2} \mathscr{M}=S^{2}$ (respectively $\left(\bar{B}_{\alpha}^{ \pm}\right)$of expansion (3.23)), or

2. the function $f_{1,0}$ of the expansion (2.6) otherwise ${ }^{11}$.

Then

1. for $k \geqq 4$ the map $\mathscr{B}_{u_{o}, k}\left(f\left(u_{o}\right)\right)\left(\right.$ respectively $\left.\overline{\mathscr{B}}_{u_{o}, k}\left(f\left(u_{o}\right)\right)\right)$ is a $C^{0}$ function of $f\left(u_{o}\right)$, and 2. for $k \geqq 6$ the map $\mathscr{B}_{u_{o}, k}\left(f\left(u_{o}\right)\right)$ (respectively $\left.\mathscr{\mathscr { B }}_{u_{o}, k}\left(f\left(u_{o}\right)\right)\right)$ is a $C^{1}$ function of $f\left(u_{o}\right)$.

The proof of Proposition 3.1 is a rather lengthy and straightforward application of the techniques developed in [8] together with parity considerations similar to those of the previous section; no details will be given. It is rather likely that the thresholds $k \geqq 4$ for continuity and $k \geqq 6$ for differentiability are not optimal; we have not attempted to analyse this question. With these thresholds it is easy to prove continuity and/or differentiability in an $L^{2}\left({ }^{2} \mathscr{M}\right)$ norm, and use interpolation to get the result for higher norms as well.

Suppose that $\stackrel{f}{f}\left(u_{o}\right)$ is such that $\mathscr{B}_{u_{o}, k}\left(f\left(u_{0}\right)\right) \in \Omega$ where, for ${ }^{2} \mathscr{M}=S^{2}, \Omega$ is the set of $\left(B_{\alpha}^{ \pm}\right)$for which $f_{\log } \not \equiv 0$ (cf. Proposition 2.4), while for ${ }^{2} \mathscr{M} \neq S^{2}$, we set $\Omega=\mathbb{R}^{N} \backslash\{0\}$. As discussed in Sect. 2, for ${ }^{2} \mathscr{M} \neq T^{2}$ the corresponding space-time will be geometrically singular at the null boundary $\mathscr{H}=\{m u=\infty\}\left(m>0\right.$ for ${ }^{2} \mathscr{M}=S^{2}, m<0$ otherwise, and if ${ }^{2} \mathscr{M} \neq S^{2}$ then we assume $\mu_{1} \neq \mathbb{N}$ ). It follows from Proposition 3.1, point 1 , that for $k \geqq 4$ the map $\mathscr{B}_{u_{o}, k}$ is continuous, so that for all $f\left(u_{o}\right)^{\text {'s in a }}$ sufficiently small neighborhood of $\stackrel{f}{f}\left(u_{o}\right)$ in $H_{4}\left({ }^{2} \mathscr{M}\right)$ the corresponding space-times will be singular at $\mathscr{H}$. Thus to prove genericity of the set of $f\left(u_{o}\right)$ 's which lead to singular $\mathscr{H}$ 's we only need to analyse what happens for $f\left(u_{o}\right)$ 's such that $\mathscr{B}_{u_{o}, k}\left(f\left(u_{o}\right)\right) \in C \Omega \equiv \mathbb{R}^{N} \backslash \Omega$.

Proposition 3.2. Suppose $k \geqq 6$, let $f(u)$ be a solution of the RT equation, $f\left(u_{o}\right) \in H_{k}\left({ }^{2} \mathscr{M}\right)$. There exists $\dot{u}_{1} \geqq u_{o}$ depending only upon $\left\|\ln f\left(u_{o}\right)\right\|_{H_{6}(2 . M)}$ such that for all $u_{1} \geqq \dot{u}_{1}$ the map $\left.\mathscr{B}_{u_{1}, k}^{\prime}\left(f\left(u_{1}\right)\right) \equiv \frac{\delta \mathscr{B}_{u_{1}, k}(\varphi)}{\delta \varphi}\right|_{\varphi=f\left(u_{1}\right)}$ is surjective. Moreover there exists $\varepsilon_{0}>0$ such that if $\left\|\ln f\left(u_{o}\right)\right\|_{H_{6}\left({ }^{2}, M\right)} \leqq \varepsilon_{0}$, then $u_{1}$ can be chosen to be equal to $u_{0}$.

Proof. If ${ }^{2} \mathscr{M} \neq S^{2}$, this is Theorem 3.1, let us thus consider the case ${ }^{2} \mathscr{M}=S^{2}$. Let $\tilde{f}(u)$ be the solution of the modified RT equation (3.1), with $\tilde{f}\left(u_{0}\right)=f\left(u_{0}\right)$. Recall that $f$ can be obtained from $\tilde{f}$ by the following procedure $[9,20]$ : let $M(u) \in S L(2, \mathbb{C})$ satisfy the equation

$$
\frac{d M}{d u}=M A(\alpha), \quad M\left(u_{o}\right)=\mathrm{id},
$$

with

for some constants $A_{b i}^{a}$, where

$$
[A(\alpha)]_{b}^{a}=\sum_{b} A_{b i}^{a} \alpha_{i}
$$

$$
\alpha_{i}=c \oint \phi_{i} \tilde{f} d \mu_{o}
$$

(cf. (3.2)). There is a natural identification between $S L(2, \mathbb{C})$ and the group of 11 Recall that $f_{1,0}$ satisfies $\Delta_{o} f_{1,0}=-\mu_{1} f_{1,0}$ and is thus determined by a finite number of
parameters $\left(B_{\alpha}\right) \in \mathbb{R}^{m_{1}}$, where $m_{1}$ is the dimension of the first non-trivial eigenspace of $\Delta_{o}$ 
conformal transformations of $S^{2}$, define $\Phi_{M}$ as the conformal map of $S^{2}$ into itself corresponding to $M \in S L(2, \mathbb{C})$, let $\Psi_{M}^{2}$ be the corresponding conformal factor,

$$
\Phi_{M}^{*} \stackrel{\circ}{g}_{a b}=\Psi_{M}^{2} \stackrel{\circ}{g}_{a b}
$$

We then have

$$
f(u)=\left[\Psi_{M(u)} \tilde{f}(u)\right] \circ \Phi_{M(u)}^{-1} .
$$

Equation (3.53) and $\lim _{u \rightarrow \infty} \tilde{f}(u)=1$ show that

$$
f(u) \circ \Phi_{M(\infty)} \times \Psi_{M(\infty)}^{-1} \stackrel{u \rightarrow \infty}{\longrightarrow} 1,
$$

thus the solution of the RT equation which has the expansion (2.8) is $f(u) \circ \Phi_{M(\infty)} \times$ $\Psi_{M(\infty)}^{-1} ;(3.53)$ gives

$$
f(u)^{\circ} \Phi_{M(\infty)} \times \Psi_{M(\infty)}^{-1}=\left[\Psi_{M(u)} \tilde{f}(u)\right]^{\circ} \Phi_{M(u)}^{-1} \circ \Phi_{M(\infty)} \times \Psi_{M(\infty)}^{-1} .
$$

Since $\alpha_{i}=O\left(e^{-\left(v^{+}+v^{-}\right) u}\right)$ (cf. (3.26)) it follows from (3.52) and from Lemma 2.1 that

$$
\begin{aligned}
|M(u)-M(\infty)| \leqq & C e^{-\left(v^{+}+v^{-}\right) u} \Rightarrow\left|\tilde{f}(u)-\tilde{f}(u)^{\circ} \Phi_{M(u)}^{-1} \circ \Phi_{M(\infty)}\right| \leqq C e^{-\left(v^{+}+v^{-}\right) u}, \\
& \left|\left(\Psi_{M(u)^{\circ}} \Phi_{M(u)^{\circ}}^{-1} \Phi_{M(\infty)}\right) \times \Psi_{M(\infty)}^{-1}-1\right| \leqq C e^{-\left(v^{+}+v^{-}\right) u},
\end{aligned}
$$

which shows that

$$
B_{\alpha}^{ \pm}=\bar{B}_{\alpha}^{ \pm} \Rightarrow \mathscr{B}_{u_{o}, k}=\overline{\mathscr{B}}_{u_{o}, k}
$$

It follows that for $u_{1} \geqq u_{o}$, the derivative $\frac{\delta \mathscr{B}_{u_{1}, k}}{\delta f}$ acting on $\varphi\left(u_{1}\right)$ is the map which to $\varphi\left(u_{1}\right)$ assigns the coefficients $\bar{B}_{\alpha}^{ \pm}$of the expansion analogous to (3.23) of solutions of the linearised equation (3.1), which is surjective by Theorem 3.1.

Theorem 3.2. Let $k \geqq 6$, suppose that $f(u), u \geqq u_{o}$ is a solution of the $\mathrm{RT}$ equation with $f\left(u_{o}\right) \in H_{k}\left({ }^{2} \mathscr{M}\right)$, let $\mathscr{B}_{u_{o}, k}\left(f\left(u_{o}\right)\right)=\left(\dot{B}_{\alpha}^{ \pm}\right) \in \mathbb{R}^{N}$.

1. There exists $u_{1} \geqq u_{0}$ and a neighborhood $\mathcal{O}_{u_{1}, k}$ of $\stackrel{\circ}{B}_{\alpha}^{ \pm}$such that for all $B_{\alpha}^{ \pm} \in \mathcal{O}_{u_{1}, k}$ there exists a solution $f_{B_{x}^{ \pm}} \in C\left(\left[u_{1}, \infty\right), H_{k}\left({ }^{2} \mathscr{M}\right)\right)$ of the $R T$ equation such that $\mathscr{B}_{u_{1}, k}\left(f_{B_{x}^{ \pm}}\left(u_{1}\right)\right)=B_{\alpha}^{ \pm}$.

2. For any $\delta_{1}>0$ there exists $\delta_{2}>0$ such that for $\left\|B_{\alpha}^{ \pm}-{\stackrel{\circ}{B_{\alpha}}}^{ \pm}\right\|_{\mathbb{R}^{N}}<\delta_{2}$ we can choose $f_{B_{\alpha}^{ \pm}}\left(u_{1}\right)$ so that $\left\|f_{B_{z}^{ \pm}}\left(u_{1}\right)-f\left(u_{1}\right)\right\|_{H_{\boldsymbol{k}^{2}(\mathscr{M})}}<\delta_{1}$.

3. There exists $\varepsilon>0$ such that if $\left\|\ln f\left(u_{o}\right)\right\|_{\left.H_{6}{ }^{2} M\right)} \leqq \varepsilon$, then $u_{1}$ can be chosen to be equal to $u_{o}$.

4. There exists $\delta_{3}>0$ such that for all $B_{\alpha}^{ \pm} \in \mathcal{O}_{u_{1}, k}$, the set of $f_{B_{2}^{ \pm}}\left(u_{1}\right)$ 's satisfying $\left\|f_{B_{z}^{ \pm}}\left(u_{1}\right)-f\left(u_{1}\right)\right\|_{H_{k^{2}(\mathscr{M})}}<\delta_{3}$ is a $C^{1}$ submanifold of finite codimension $N(N=12$ if $\left.{ }^{2} M=S^{2}\right)$ of $H_{k}\left({ }^{2} \mathscr{M}\right)$.

Proof. It follows from Proposition 3.1 that for $u_{1} \geqq u_{o}, \mathscr{B}_{u_{1}, k}: H_{k}\left({ }^{2} \mathscr{M}\right) \rightarrow \mathbb{R}^{N}$ is differentiable, thus both $\operatorname{Ker} \mathscr{B}_{u_{1}, k}^{\prime}$ and $\left(\operatorname{Ker} \mathscr{B}_{u_{1}, k}\right)^{\perp}$ are (closed) Banach spaces, and the map $\mathscr{F}:\left(\operatorname{Ker} \mathscr{B}_{u_{1}, k}^{\prime}\right)^{\perp} \rightarrow \mathbb{R}^{N}$ defined by $\mathscr{F}(\varphi)=\mathscr{B}_{u_{1}, k}(\varphi)$ is differentiable. We have $\mathscr{F}^{\prime} \equiv \frac{\delta \mathscr{F}}{\delta \varphi}=\left.\mathscr{B}_{u_{1}, k}^{\prime}\right|_{\left(\mathrm{Ker} \mathscr{G}_{u_{1}, k}^{\prime}\right.}{ }^{\perp}$, and Proposition 3.2 shows that $\mathscr{F}^{\prime}$ is an isomorphism.

By the implicit function theorem, $\mathscr{F}$ is an isomorphism from $\mathscr{U}_{u_{1}, k} \subset\left(\operatorname{Ker} \mathscr{B}_{u_{1}, k}\right)^{\perp}$ 
to an open neighbourhood $\mathcal{O}_{u_{1}, k}$ of $\left(\stackrel{\circ}{*}_{\alpha}^{ \pm}\right)$, which proves points $1-3$. Point 4 follows from the implicit mapping theorem, cf. e.g. [15 Chapter I, §5].

Corollary 3.1. 1. Let ${ }^{2} \mathscr{M}=S^{2}$. There exist non-trivial (i.e. $f \not \equiv f_{\infty}$, where $f_{\infty}$ is a conformal factor for a conformal transformation) RT metrics such that $\left|f-f_{\infty}\right| \leqq$ $C e^{-15 v^{+} u}=C e^{-30 u / m}$. Every such metric can be $C^{557}$ extended across $\mathscr{H}$.

2. Let ${ }^{2} \mathscr{M} \neq S^{2}$. There exist non-trivial (i.e. $f \neq 1$ ) RT metrics such that $|f-1| \leqq$ $C e^{-\lambda_{2} u}, \lambda_{2}=\mu_{2}\left(\mu_{2}+R_{o}\right) /(12 m)$, where $\mu_{2}$ is the second non-trivial eigenvalue of $-\Delta_{o}$.

Proof. We shall consider the case ${ }^{2} \mathscr{M}=S^{2}$ only, the remaining cases follow in a similar manner. Let $u_{o} \in \mathbb{R}$ be arbitrary, let $f\left(u_{o}\right)=1$, thus the corresponding RT space-time is the Schwarzschild space-time; for any $k \geqq 6$ it follows from Theorem 3.2 , point 4 , that there exists a submanifold of codimension 12 of $f\left(u_{o}\right)$ 's in $H_{k}\left({ }^{2} \mathscr{M}\right)$ for which $\mathscr{B}_{u_{o}, k}\left(f\left(u_{o}\right)\right)=0$, and the arguments of the proof of Lemma 2.1 show that for such initial data we will have $\left|f-f_{\infty}\right| \leqq C e^{-15 v^{+} u}$, for some $f_{\infty}=\lim _{u \rightarrow \infty} f(u)$. The $C^{557}$ extendability follows from Lemma 2.1 , point 3 , by parity considerations.

Proof of Theorem 2.2. Let $X_{k}=\left\{\AA \in f \in H_{k}\left({ }^{2} \mathscr{M}\right)\right.$ : the horizon of the RT space-time with $f$ such that $f\left(u_{0}\right)=f$ is singular $\}$. For $k \geqq 4$ openness of $X_{k}$ follows from Proposition 3.1, point 1. To prove density, consider a solution $f(u)$ of the RT equation such that $\left\|\ln f\left(u_{o}\right)\right\|_{H_{6}\left({ }^{2} \mathscr{M}\right)}<\varepsilon, \varepsilon$ given by Theorem 3.2, point 3 , and assume that $f\left(u_{o}\right) \notin X_{k}$. Let $\left(\stackrel{\circ}{B}_{\alpha}^{ \pm}\right)=\mathscr{B}_{u_{o}, k}\left(f\left(u_{o}\right)\right)$, thus $\left(\dot{B}_{\alpha}^{ \pm}\right) \in C \Omega$, where $\Omega=\left\{B_{\alpha}^{ \pm}: f_{\log } \not \equiv 0\right\}$ if ${ }^{2} \mathscr{M}=S^{2}$, and $\Omega=$ $\mathbb{R}^{N} \backslash\{0\}$ otherwise. Since $\Omega$ is dense in $\mathbb{R}^{N}$ (cf. Proposition 2.2 if ${ }^{2} \mathscr{M}=S^{2}$ ), it follows that there exists a sequence $B_{\alpha, i}^{ \pm} \stackrel{i \rightarrow \infty}{\longrightarrow} \stackrel{\circ}{\alpha}_{\alpha}^{ \pm}$, such that $B_{\alpha, i}^{ \pm} \in \Omega$; for $k \geqq 6$ it follows from Theorem 3.2, point 2 , that there exists a sequence $f_{i}\left(u_{o}\right) \in H_{k}\left({ }^{2} \mathscr{M}\right)$ converging to $f\left(u_{o}\right)$ in $H_{k}\left({ }^{2} \mathscr{M}\right)$ norm such that $\mathscr{B}_{u_{o}, k}\left(f_{o}\left(u_{o}\right)\right)=B_{\alpha, i}^{ \pm}$, and thus the corresponding spacetimes are singular at $\mathscr{H}$.

Proof of Theorem 2.1. Let $X=\left\{\stackrel{\circ}{f} \in C^{\infty}\left({ }^{2} \mathscr{M}\right)\right.$ : the horizon $\mathscr{H}$ of the RT space-time with $f$ such that $f\left(u_{o}\right)=\stackrel{\circ}{f}$ is singular $\}$. Openness follows as in the proof of Theorem 2.2; to show density consider a solution $f(u)$ of the RT equation such that $f\left(u_{o}\right) \notin X$, and $\left\|\ln f\left(u_{o}\right)\right\|_{H_{6}\left({ }^{2} \mathscr{M}\right)}<\varepsilon, \varepsilon$ given by Theorem 3.2, point 3 . For any $k \in \mathbb{N}$ by Theorem 3.2 point 2 we can find a sequence $f_{i}^{k} \in H_{\max (k+2,6)}\left({ }^{2} \mathscr{M}\right)$ converging to $f\left(u_{o}\right)$ in $H_{\max (k+2,6)}\left({ }^{2} \mathscr{M}\right)$ norm such that $f_{i}^{k} \in X$. By density of $C^{\infty}\left({ }^{2} \mathscr{M}\right)$ in $H_{\max (k+2,6)}\left({ }^{2} \mathscr{M}\right)$ there exists a sequence $f_{i, j}^{k} \in C^{\infty}\left({ }^{2} \mathscr{M}\right)$ such that $f_{i, j}^{k} \stackrel{j \rightarrow \infty}{\longrightarrow} f_{i}^{k}$ in $H_{\max (k+2,6)}\left({ }^{2} \mathscr{M}\right)$ norm; by continuity of $\mathscr{B}_{u_{o}, \max (k+2,6)}$ it follows that for $j \geqq j(i), \mathscr{B}_{u_{o}, \max (k+2,6)}\left(f_{i, j}^{k}\right) \in \Omega$, and thus for $j \geqq j(i)$ we have $f_{i, j}^{k} \in X$. We can find a sequence $j_{i} \geqq j(i)$ such that $f_{i, j_{i}}^{k} \stackrel{i \rightarrow \infty}{\longrightarrow} f\left(u_{o}\right)$ in $H_{\max (k+2,6)}\left({ }^{2} \mathscr{M}\right)$ norm, thus $f_{i, j_{i}}^{k} \stackrel{\stackrel{i}{\longrightarrow} \longrightarrow \infty}{\longrightarrow} f\left(u_{o}\right)$ in $C^{k}\left({ }^{2} \mathscr{M}\right)$ norm by Sobolev embedding; redefine $f_{i}^{k}$ to be $f_{i, j_{i}}^{k}$. Define a sub-sequence $f_{i_{l}}^{k}$ of $f_{i}^{k}$ by the condition $\left\|f_{i_{l}}^{k}-f\left(u_{o}\right)\right\|_{C^{k}(2, \mathscr{M})} \leqq 2^{-(l+k)}$. The sequence $f_{i_{l}}^{l}$ converges to $f\left(u_{o}\right)$ in $C^{\infty}$ topology as $l$ tends to infinity, and the solutions $f_{l}(u)$ of the RT equation satisfying $f_{l}\left(u_{o}\right)=f_{i_{l}}^{l}$ are singular on the event horizon $\mathscr{H}$.

\section{The Robinson-Trautman Equation as a Dynamical System on the Space of Metrics}

Let Riemm $\left({ }^{2} \mathscr{M}\right)$ be the space of smooth metrics on a compact, connected, orientable two dimensional manifold ${ }^{2} \mathscr{M}$, let $\operatorname{Diff}_{o}\left({ }^{2} \mathscr{M}\right)$ be the connected component of the 
space of smooth diffeomorphisms of ${ }^{2} \mathscr{M}$ which contains the identity, both equipped with the $C^{\infty}$ topology. The RT equation with, say, $12 \mathrm{~m}=1$, may be used to define ${ }^{12}$ a flow $\Phi_{u}: \operatorname{Riemm}\left({ }^{2} \mathscr{M}\right) / \operatorname{Diff}_{o}\left({ }^{2} \mathscr{M}\right) \rightarrow \operatorname{Riemm}\left({ }^{2} \mathscr{M}\right) / \operatorname{Diff}_{o}\left({ }^{2} \mathscr{M}\right)$ as follows: let $[g] \epsilon$ Riemm $\left({ }^{2} \mathscr{M}\right) / \operatorname{Diff}_{o}\left({ }^{2} \mathscr{M}\right)$, let $g(u)$ be the solution of the RT equation such that $g(0)=g$, then $\Phi_{u}([g])=[g(u)]$ (throughout this section we use $[g]$ to denote the equivalence class of $g$ in Riemm $\left.\left({ }^{2} \mathscr{M}\right) / \operatorname{Diff}_{o}\left({ }^{2} \mathscr{M}\right)\right)$. If we define $\operatorname{Riemm}_{o}\left({ }^{2} \mathscr{M}\right)$ as the set of metrics $\stackrel{\circ}{g}_{a b}$ for which $R_{o} \equiv R\left(\stackrel{\circ}{g}_{a b}\right) \in\{-2,0,2\}$, then the results of [8] show that $\Phi_{\infty}=$ $\lim _{u \rightarrow \infty} \Phi(u)$ exists, and that $\mathscr{T}\left({ }^{2} \mathscr{M}\right) \equiv \operatorname{Riemm}_{o}\left({ }^{2} \mathscr{M}\right) / \operatorname{Diff}_{o}\left({ }^{2} \mathscr{M}\right)$ is an attractor for $\Phi_{u}$, with basin of attraction equal to the whole of $\operatorname{Riemm}\left({ }^{2} \mathscr{M}\right) / \operatorname{Diff}_{o}\left({ }^{2} \mathscr{M}\right)$. Given $\left[g_{o}\right] \in \mathscr{T}\left({ }^{2} \mathscr{M}\right)$ let $\mathscr{A}\left(\left[g_{o}\right],{ }^{2} \mathscr{M}\right)$ be the basin of attraction of $\left[g_{o}\right]$ :

$$
\mathscr{A}\left(\left[g_{o}\right],{ }^{2} \mathscr{M}\right)=\left\{[g] \in \operatorname{Riemm}\left({ }^{2} \mathscr{M}\right) / \operatorname{Diff}_{o}\left({ }^{2} \mathscr{M}\right): \Phi_{\infty}([g])=\left[g_{o}\right]\right\} .
$$

We have

$$
\begin{gathered}
\bigcup_{\left[g_{o}\right] \in \mathscr{T}\left({ }^{2} \mathscr{M}\right)} \mathscr{A}\left(\left[g_{o}\right],{ }^{2} \mathscr{M}\right)=\operatorname{Riemm}\left({ }^{2} \mathscr{M}\right) / \operatorname{Diff}_{o}\left({ }^{2} \mathscr{M}\right), \\
\forall\left[g_{o}\right] \in \mathscr{T}\left({ }^{2} \mathscr{M}\right) \quad \mathscr{A}\left(\left[g_{o}\right],{ }^{2} \mathscr{M}\right) \neq \varnothing .
\end{gathered}
$$

We wish to point out that because of the asymptotic expansion (2.6) one can naturally associate to $\mathscr{T}\left({ }^{2} \mathscr{M}\right)$ an infinite sequence of "blow-up" structures, as follows: let $\left[g_{o}\right] \in \mathscr{T}\left({ }^{2} \mathscr{M}\right)$, let $\left\{\mu_{i}\right\}_{i \geqq 1}$ be the increasingly ordered spectrum of $-\Delta_{o}$, $\mu_{1}>0$, let $\mathscr{H}_{i} \subset L^{2}\left({ }^{2} \mathscr{M}\right)$ be the $i^{\text {th }}$ eigenspace; we have $\mathscr{H}_{i} \approx \mathbb{R}^{m_{\mathbf{t}}}$ for some $m_{i}$. Define $\left\{\lambda_{i}\right\}=\left\{\mu_{i}\left(\mu_{i}-2\right)\right\}_{i \geqq 2}$ if ${ }^{2} \mathscr{M}=S^{2}$; and $\left\{\lambda_{i}\right\}=\left\{\mu_{i}\left(\mu_{i}+R_{o}\right)\right\}_{i \geqq 1}$ otherwise (thus $\left\{\lambda_{i}\right\}$ is the spectrum of the operator which appears at the right-hand side of the linearization at a metric $g_{o} \in \operatorname{Riemm}{ }_{o}\left({ }^{2} \mathscr{M}\right)$ of the RT $\left({ }^{2} \mathscr{M} \neq S^{2}\right)$ or of the modified RT $\left({ }^{2} \mathscr{M}=S^{2}\right)$ equation). Let $\left\{v_{i}\right\}_{i \geqq 1}$ be as described in Sect. 2 (cf. [9] for more details). We have $\left\{\lambda_{i}\right\} \subset\left\{v_{i}\right\}$, and along the lines of the proof of Lemma 2.1 one shows, that if we write

$$
f \sim 1+\sum_{i=1}^{\infty} \sum_{j=0}^{N(i)} f_{i, j} u^{j} e^{-v_{i} u},
$$

where " " stands for "asymptotic to," in the sense of Eqs. (2.6), (2.7), then

1. if $v_{i} \notin\left\{\lambda_{l}\right\}_{l \in \mathbb{N}}$, then the functions $f_{i, j}, j=0, \ldots, N(i)$ are defined uniquely by the functions $f_{k, 0}, 1 \leqq k \leqq i-1$,

2. if $v_{i}=\lambda_{l}$ for some $\bar{l}$, then the functions $f_{i, j}, j=1, \ldots, N(i)$ are defined uniquely by the functions $f_{k, 0}, 1 \leqq k \leqq i-1$, and there exists a function $\phi_{i}$ determined uniquely by $f_{k, 0}, 1 \leqq k \leqq i-1$, such that $f_{i}-\phi_{i} \in \mathscr{H}_{i}$.

The coefficients $X_{i}=\left(X_{i, k}\right)_{k=1}^{m_{i}} \in \mathbb{R}^{m_{i}}$ of the decomposition $f_{i}-\phi_{i}=\sum_{k=1}^{m_{i}} X_{i, k} \phi_{i, k}$, where the functions $\phi_{i, k}$ form a basis of $\mathscr{H}_{i}$, will be called the free coefficients of the expansion (4.3).

\footnotetext{
${ }^{12}$ Since $\operatorname{Riemm}\left({ }^{2} \mathscr{M}\right) / \operatorname{Diff}_{o}\left({ }^{2} \mathscr{M}\right)$ is a stratified Inverse-Limit-Hilbert manifold [3] with singularities occurring on sets of metrics for which the isometry group jumps, and since the symmetries of a metric are preserved by the RT equation, it seems likely that one can define the RT equation on $\operatorname{Riemm}\left({ }^{2} \mathscr{M}\right) / \operatorname{Diff}_{o}\left({ }^{2} \mathscr{M}\right)$; a simpler way, which avoids some technicalities, is to proceed as above
} 
Let $k \in \mathbb{N}$, we have $\bigoplus_{i=1}^{k} \mathscr{H}_{i}=\mathbb{R}^{\boldsymbol{M}_{k}}, M_{k}=\sum_{i=1}^{k} m_{i}$. For any $X \in \mathbb{R}^{\boldsymbol{M}_{k}}$ we define

$$
\begin{aligned}
\mathscr{A}_{k}\left(X,\left[g_{o}\right],{ }^{2} \mathscr{M}\right)=\{ & \left\{[g] \in \mathscr{A}\left(\left[g_{o}\right],{ }^{2} \mathscr{M}\right):\right. \text { the free coefficients } \\
& \text { of the expansion (4.3) are equal to } X\} .
\end{aligned}
$$

We have

$$
\bigcup_{X \in \mathbb{R}^{M_{k}}} \mathscr{A}_{k}\left(X,\left[g_{o}\right],{ }^{2} \mathscr{M}\right)=\mathscr{A}\left(\left[g_{o}\right],{ }^{2} \mathscr{M}\right)
$$

and more generally, if we set $\bigoplus_{i=1}^{l} \mathscr{H}_{i}=\mathbb{R}^{M_{k}} \oplus \mathbb{R}^{M_{l}-M_{k}}$, where $\mathbb{R}^{M_{l}-M_{k}}=\bigoplus_{i=k+1}^{l} \mathscr{H}_{i}$, then

$$
\bigcup_{X_{2} \in \mathbb{R}^{M_{l}-M_{k}}} \mathscr{A}_{l}\left(\left(X_{1}, X_{2}\right),\left[g_{o}\right],{ }^{2} \mathscr{M}\right)=\mathscr{A}_{k}\left(X_{1},\left[g_{o}\right],{ }^{2} \mathscr{M}\right) .
$$

Note that if $[g] \in \mathscr{A}_{l}\left(\left(0, X_{2}\right),\left[g_{o}\right],{ }^{2} \mathscr{M}\right), X_{2} \in \mathbb{R}^{M_{l}-M_{k}}$, then $\Phi_{u}([g)]$ converges to $\Phi_{\infty}([g])$ exponentially fast with decay rate larger than or equal to $\lambda_{k+1}$.

The terminology introduced above allows us to restate what has been proved in Sect. 3 as follows:

Proposition 4.1. For any $g_{o} \in \operatorname{Riemm}_{o}\left({ }^{2} \mathscr{M}\right)$ there exists $\varepsilon_{o}>0$ such that if $X \in \mathbb{R}^{M_{1}}$, $\|X\|_{\mathbb{R}^{M_{1}}}<\varepsilon_{o}$, then

$$
\mathscr{A}_{1}\left(X,\left[g_{o}\right],{ }^{2} \mathscr{M}\right) \neq \varnothing .
$$

Proposition 4.2. Let ${ }^{2} \mathscr{M}=S^{2}$. For any $g_{o} \in \operatorname{Riemm}_{o}\left({ }^{2} \mathscr{M}\right)$ there exists $\varepsilon_{o}>0$ such that if $X \in \mathbb{R}^{M_{2}},\|X\|_{\mathbb{R}^{M_{2}}}<\varepsilon_{o}$, then

$$
\mathscr{A}_{2}\left(X,\left[g_{o}\right],{ }^{2} \mathscr{M}\right) \neq \varnothing .
$$

If rather than considering smooth metrics and diffeomorphisms we consider $H_{l}$ metrics and $H_{l+1}$ diffeomorphisms, the slice theorem [10] and Theorem 3.2 give:

Theorem 4.1. Let $g_{o} \in \operatorname{Riemm}_{o}\left({ }^{2} \mathscr{M}\right)$ and suppose that the isometry group of $g_{o}$ is trivial. For $l \geqq 6$ there exists a neighbourhood $\mathcal{O}_{l}$ of $\left[g_{o}\right]$ (in the $H_{l}$ quotient topology) such that $\mathscr{A}_{1}\left(X,\left[g_{o}\right],{ }^{2} \mathscr{M}\right) \cap \mathcal{O}_{l}$ is a $C^{1}$ submanifold of finite codimension.

It seems natural to ask the question:

$$
\left\{X: \mathscr{A}_{k}\left(X,\left[g_{o}\right],{ }^{2} \mathscr{M}\right)=\varnothing\right\} \stackrel{?}{=} \varnothing .
$$

Define $X_{\infty}$ " $\in \mathbb{R}^{\infty}$ " as a sequence $X_{i} \in \mathbb{R}^{M_{i}}$ such that $P_{\mathbb{R}^{M}} X_{i}=X_{j}$ for $j \leqq i$, where $P_{\mathbb{R}^{M_{j}}}$ is thecoordinate projection on $\mathbb{R}^{M_{j}}=\mathbb{R}^{M_{J}} \oplus\{0\} \subset \mathbb{R}^{M_{j}} \oplus \mathbb{R}^{M_{i}-M_{J}}=\mathbb{R}^{M_{i}}$; set

$$
\mathscr{A}_{\infty}\left(X_{\infty},\left[g_{o}\right],{ }^{2} \mathscr{M}\right)=\bigcap_{k} \mathscr{A}_{k}\left(X_{k},\left[g_{o}\right],{ }^{2} \mathscr{M}\right) .
$$

The conjecture mentioned at the end of Sect. 2.1 can be formulated as follows:

$$
\mathscr{A}_{\infty}\left(X_{\infty},\left[g_{o}\right],{ }^{2} \mathscr{M}\right) \stackrel{?}{=}\{\text { a one dimensional submanifold, unless empty }\} .
$$

Acknowledgements. The authors acknowledge useful discussions with R. Bartnik, C. Hodgson and G. Latham. We are grateful to C. McIntosh and to the members of the Relativity Group at Monash University, Melbourne, for giving us access to their computer facilities where most 
of our CPU time-consuming computer symbolic algebraic manipulations were performed. One of us (P.T.C.) wishes to thank the Mathematische Forschungsinstitut, Oberwolfach, and the Max Planck Institut für Astrophysik, Garching, for hospitality during the final stage of work on this paper.

\section{References}

1. Abramowitz, M., Stegun, A. I.: Handbook of Mathematical Functions, pp. 331-341. Washington, DC: US National Bureau of Standards, Chap. 8, 1965

2. Bičák, J., Schmidt, B.: Phys. Rev. D40, 1872 (1989)

3. Bourguignon, J.-P.: Comp. Math. 30, 1 (1975); Fischer, A.: The Theory of Superspace. In: Relativity: Proceedings of the Relativity Conference in the Midwest, Carmeli, M., Fickler, S. I., Witten, L. (eds.). New York: Plenum Press 1970

4. Chavel, I.: Eigenvalues in Riemannian Geometry. New York: Academic Press 1984

5. Christodoulou, D.: Commun. Math. Phys. 105, 337 (1986), 106, 587 (1986), 109, 591 (1987); 109, 613 (1987)

6. Christodoulou, D.: Commun Pure Appl. Math. 44, 339 (1991)

7. Christodoulou, D., Klainerman, S.: The Global Nonlinear Stability of Minkowski Space, preprint (1989)

8. Chruściel, P. T.: Commun. Math. Phys. 137, 289 (1991)

9. Chruściel, P. T.: On the global structure of Robinson-Trautman space-times, preprint CMA-R23-90. Proc. R. Soc. London (in press)

10. Ebin, D.: Proc. Symp. Pure Math. A.M.S. 15, 11 (1970)

11. Fischer, A., Tromba, A.: Math. Ann. 237, 311 (1984)

12. Foster, J.: Proc. Camb. Phil. Soc. 66, 521 (1969)

13. Friedrich, H.: Commun. Math. Phys. 107, 587 (1986)

14. Jenni, F.: Comment. Math. Helv. 59, 193 (1984)

15. Lang, S.: Differential Manifolds. Reading, MA: Addison Wesley 1972

16. Rendall, A.: Class. Quantum Grav. 5, 1339 (1988)

17. Robinson, I., Trautman, T.: Proc. Roy. Soc. Lond. A265, 463 (1962)

18. Schmidt, B.: Gen. Rel. Grav. 20, 65 (1988)

19. Singleton, D.: PhD Thesis, Monash University, Melbourne 1989

20. Singleton, D.: Class. Quantum Grav. 7, 1333 (1990)

21. Tod, P.: Class. Quantum Grav. 6, 1159 (1989)

Communicated by S.-T. Yau 